\title{
Oral delivery system for low molecular weight protamine-dextran-poly(lactic-co-glycolic acid) carrying exenatide to overcome the mucus barrier and improve intestinal targeting efficiency
}

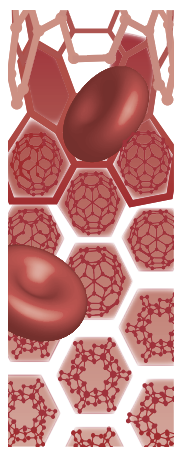

\author{
Yina Song ${ }^{\ddagger}, 1$ Yanan Shi ${ }^{\star \ddagger, 2}$, Liping Zhang ${ }^{1}$, Haiyan $\mathrm{Hu}^{1}$, Chunyan Zhang ${ }^{1}$, Miaomiao Yin ${ }^{1}$, \\ Xuemei Zhang ${ }^{3}$ \& Kaoxiang Sun $* *, 1,3$ \\ ${ }^{1}$ School of Pharmacy, Key Laboratory of Molecular Pharmacology \& Drug Evaluation (Yantai University), Ministry of Education, \\ Collaborative Innovation Center of Advanced Drug Delivery System \& Biotech Drugs in Universities of Shandong, Yantai University, \\ Yantai, 264005, PR China \\ ${ }^{2}$ School of Pharmacy, Binzhou Medical University, Yantai, 264005, PR China \\ ${ }^{3}$ State Key Laboratory of Long-Acting \& Targeting Drug Delivery System, Luye Pharmaceutical Co. Ltd, Yantai, 264005, PR China \\ *Author for correspondence: Tel.: +86 1876645 3922; Fax: +86 535394 6187; shiyanan001@163.com \\ **Author for correspondence: Tel.: +86 535380 8266; Fax: +86 535670 6066; sunkaoxiang@luye.com \\ $\ddagger$ Authors contributed equally
}

\begin{abstract}
Aim: This study aimed to explore the effect of nanoparticles loaded with exenatide in overcoming the mucus barrier and improving intestinal targeting efficiency, to improve the oral bioavailability. Materials \& methods: Low molecular weight protamine (LMWP)-dextran-poly(lactic-co-glycolic acid) was used to create LMWP-dextran-poly(lactic-co-glycolic acid)-nanoparticles (LDPs) encapsulating exenatide-Zn ${ }^{2+}$ complex.Results \& conclusion: LDPs showed improved penetration of the mucus barrier, and LMWP was helpful for mediating cell translocation through protein transduction domains. The absorption sites and distribution rates of LDPs were verified by intestinal localization experiments and in vivo distribution experiments. Cell uptake and transmembrane experiments confirmed the absorption efficiency in the intestinal epithelium. Furthermore, the relative bioavailability after oral administration of exenatide-Zn ${ }^{2+}$-LDPs was $8.4 \%$, with a significant hypoglycemic effect on Type 2 diabetic mice.
\end{abstract}

First draft submitted: 3 September 2018; Accepted for publication: 3 January 2019; Published online:

22 March 2019

Keywords: exenatide-Zn² $\bullet$ intestinal absorption $\bullet$ LMWP-DEX-PLGA • mucus penetration • oral delivery • targeted nanoparticle

The number of peptide drugs in preclinical development and undergoing clinical trials has increased in recent years [1]. This is because proteins and peptides have advantages of high efficiency, low toxicity, good selectivity and good tolerance among other properties. However, at present, protein and polypeptide drugs are mainly administered by frequent injections, leading to poor patient compliance [2]. Therefore, development of strategies, which can make oral peptide administration feasible, has become a primary goal in the field of biopharmaceuticals [3]. The possibility of oral peptide delivery is particularly critical for drugs used in the treatment of chronic diseases. In the specific case of antidiabetic drugs like insulin and glucagon-like peptide (GLP-1), oral administration has the additional advantage of mimicking normal physiological pathways such as hepatic portal circulation [4]. In fact, insulin transported through the intestinal epithelium directly reaches the liver as the target organ for pancreatic secretion of insulin. In contrast, when insulin is injected into the whole body, only a limited amount is effective at its target site. Oral delivery of proteins and peptides has attracted great interest in academic research and the

Future Medicine 
pharmaceutical industry over the past decade [3,5]. During this period, different types of nanoparticles (NPs) have been extensively developed to improve oral absorption of protein and peptide drugs [5,6].

Degradation by gastrointestinal (GI) enzymes and poor penetration of the intestinal mucosa are the main obstacles for absorption of peptide drugs $[7,8]$. A variety of strategies to provide structural protection and improve absorption of peptide drugs have been reported. These include use of enzyme inhibitors [9], absorption enhancers [3], chemical modifications and encapsulation of peptide drugs within microspheres [10], and NPs. Some of these nanocarriers were shown to protect peptides against degradation by intestinal enzymes [11]. However, the development of mucus-diffusive and percutaneous NPs remains a key challenge in the field of oral peptide delivery $[12,13]$.

Based on this background, we developed a nanometer carrier, which can protect peptide drugs against degradation by GI enzymes, penetrate the mucous layer and be ingested by the intestinal epithelium, and clarified its uptake mechanism.

Exenatide-zinc freeze-dried complex (exenatide- $\mathrm{Zn}^{2+}$ ) was selected as the model drug. Exenatide has 53\% homology to human GLP-1 and can reduce elevated blood glucose levels above the normal range. Exenatide is a novel Type 2 diabetes drug and the only drug that can enable Type 2 diabetes patients to simultaneously control their blood sugar and body weight [14]. It was reported that $\mathrm{Zn}^{2+}$ can improve the thermal stability and function of selected proteins through coordination of the imidazole in the active site, thereby improving their activity [15]. Specific target NPs were prepared by encapsulating exenatide- $\mathrm{Zn}^{2+}$ in low molecular weight protamine-dextranpoly(lactic-co-glycolic acid) (LMWP-DEX-PLGA) as a polymer material by a multiple emulsion method.

LMWP with sequence VSRRRRRRGGRRRRC prepared by enzymatic hydrolysis of natural protamine is a strong and nontoxic cell-penetrating peptide, and by far the most potent protein transduction domain (PTD) peptide [16-18]. LMWP is as effective as TAT protein, the classic HIV transcriptional activator that acts as an effective delivery system for cell entry without antigenicity and mutagenicity, and is considered safe and nontoxic by the US FDA. PTD-mediated cell translocation does not resemble any existing transcellular pathways, including the frequently used receptor-mediated endocytosis [16]. The attraction of this system for potential biomedical applications is that PTD-mediated cell internalization does not cause membrane disturbance or damage [19]. Furthermore, all cell types are susceptible to PTD-mediated cell transduction.

DEX, a colloidal and hydrophilic polymer, is considered to be one of the most useful agents for binding of bioactive substances in targeted drug delivery systems. DEX used in the preparation of oral NPs has many advantages, including abundant hydroxyl groups favorable for chemical modification [20], a peptide-friendly hydrophilic segment for improved drug encapsulation rate and enhanced interactions between NPs and the GI tract [21], and low toxicity and immunogenicity for reduced in vivo immunogenicity and therapeutic drug delivery [22]. For the delivery system, penetration is achieved by the interactions of positively charged DEX with negative charges on the cell membrane. An ideal delivery system for oral administration of a macromolecular polypeptide should prolong the intestinal residence time, reversibly increase the permeability of the mucosal epithelium to enhance the absorption and provide the complete macromolecular polypeptide to the systemic circulation [23]. It was reported that hydrophilic and electrically neutral surfaces can be used as mucus-passivating layers to protect particles against capture, which is conducive to NP passage through mucous substrates. It was also reported that DEX-PLGA copolymers have amphiphilic properties and can form core-shell NPs as carriers [12]. PLGA, an FDA-approved polymer with good biocompatibility, safety and biodegradability, can prolong the residence time of compounds in the blood [24].

Therefore, the purpose of this study was to explore the effect of LMWP-DEX-PLGA-NPs (LDPs) loaded with exenatide for overcoming the mucus barrier and improving the intestinal targeting efficiency, with a view to improving the oral bioavailability of macromolecular protein drugs. The mechanism is shown in Figure 1. The first step is penetration of the mucus layer, which occurs because that the globally electrically neutral NPs can easily penetrate the mucus layer, while positively charged particles have stronger mucosal adhesion. Moreover, the surface modification of NPs by DEX makes them hydrophilic and allows easy penetration of the mucus layer of the small intestine [25]. The next step is infiltration of the intestinal epithelium, which consists of three pathways. The first pathway is paracellular transport. Positively charged NPs can open tight junctions between the small intestinal epithelium and improve paracellular permeability, because DEX provides the periphery of nanocrystals with weak electrical positivity [26]. Furthermore, drug transport through intercellular spaces is small compared with transmembrane transport [27]. The second pathway is the M-cell transport pathway. M-cells have few cytoplasmic lysosomes and many mitochondria. They are primarily responsible for ingesting antigens and other microorganisms from the intestinal cavity and transporting them to the mucosal immune system [28]. The third pathway is transcellular transport mediated by PTDs. Because of the existence of LMWP, PTD-mediated 


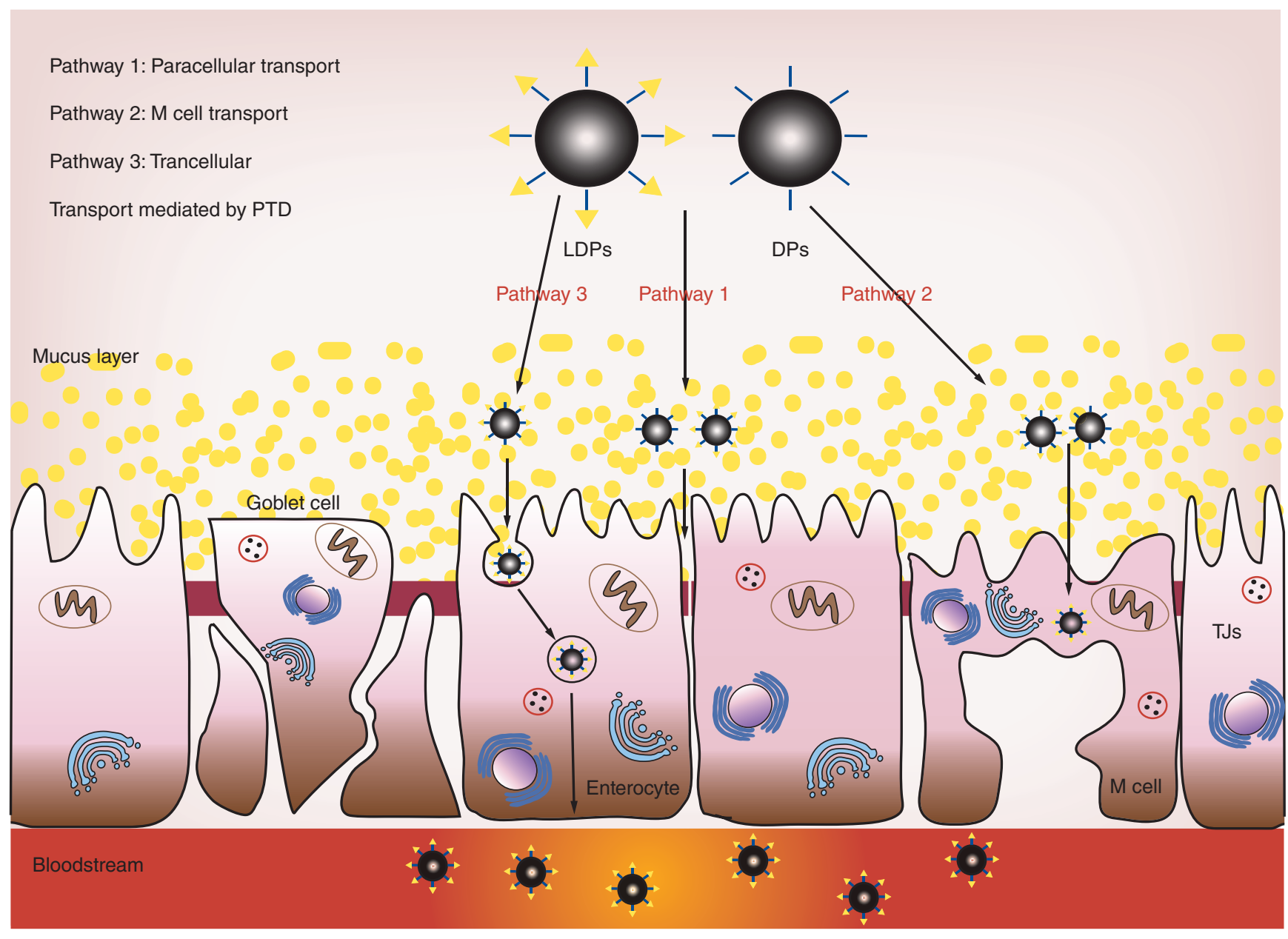

Figure 1. Schematic illustration for transepithelial transport of low molecular weight protamine-dextran-poly(lactic-co-glycolic acid)-nanoparticles to overcome multiple barriers of the intestinal epithelium by exploiting the protein transduction domainmediated pathway.

DP: Dextran-poly(lactic-co-glycolic acid)-nanoparticles; LDP: Low molecular weight protamine-dextran-poly(lactic-co-glycolic acid)-nanoparticles; PTD: Poly transduction domain.

cell translocation occurs. Thus, this pathway is only active for LDPs [29]. Because enterocytes comprise the largest proportion of small intestinal epithelial cells, both DEX-PLGA-NPs (DPs) and LDPs are involved in the tight junction pathway and M-cell pathway. The impact of DPs and LDPs on tight junctions has not been confirmed. It is necessary to carry out the research in this field at a later stage. As a result, LDPs eventually enter the systemic circulation in greater quantities than DPs. To confirm the in vitro-in vivo correlation, absorption of LDPs into the intestinal villi was visualized by confocal laser scanning microscopy. The results revealed that transport of LDPs was mediated by PTDs, but transport of DPs was not. The specific transcellular transport pathway of DPs was not studied in this paper. Compared with the other two transport pathways, whether the M-cell pathway has a significant effect on oral administration remains to be further studied. An in vitro cell monolayer model as a simulated intestinal epithelial mucosal barrier was used to further verify the mechanism of NPs. Finally, the pharmacological activities of LDPs were examined in healthy rats and Type 2 diabetic mice after oral administration.

\section{Materials \& methods}

\section{Materials}

LMWP (VSRRRRRRGGRRRRC) was purchased from Shanghai Xinhao Biotechnology Co. Ltd. Exenatide was purchased from Shanghai GL Biochem Ltd (Shanghai, China). Phosphate-buffered saline (PBS) was purchased from 
Fuzhou Maixin Biotech (Beijing, China). An exenatide-4 ELISA kit was purchased from Phoenix Pharmaceutics, Inc. (CA, USA). Dialysis bags (retained molecular weight range: 8000-14000) were purchased from Beijing Solarbio Science \& Technology Co. Ltd (Beijing, China). DEX-PLGA and LMWP-DEX-PLGA were from Xi'an Ruixi Biopharmaceutical Co. Ltd (Xi'an, China). Nile red was purchased from Aladdin Industrial (Shanghai, China). Methyl- $\beta$-cyclodextrin $(\mathrm{M}-\beta-\mathrm{CD})$ and chlorpromazine hydrochloride were purchased from Shanghai Aladdin Reagent Co. Ltd (Shanghai, China). Acetonitrile was purchased from Yantai Yuansheng Chemical Laboratory Equipment Co. Ltd (Shandong, China). Dichloromethane was purchased from Chinese Medicines Group Chemical Reagent Co. Ltd (Beijing, China). Hoechst 33342 were purchased from Beijing Fanbo Biochemical Co., Ltd (Beijing, China). DMEM was purchased from Thermo Fisher Scientific (MA, USA).

\section{Preparation \& characterization of NPs Preparation of LDPs \& DPs}

LMWP-DEX-PLGA $(20 \mathrm{mg})$ was added to DMSO $(0.33 \mathrm{ml})$ and shaken until completely dissolved. Dichloromethane $(0.67 \mathrm{ml})$ was then added to the DMSO solution to become an oil phase after shaking and mixing. Exenatide- $\mathrm{Zn}^{2+}(2 \mathrm{mg})$ was added to the oil phase, and a colostrum was obtained by ultrasonication for $30 \mathrm{~s}$ (10-s bursts, 5-s intervals) using an ultrasonic cell pulverizer (JY92-2D, Xinzhi, Ningbo, China). The composite emulsion was dispersed into polyvinyl alcohol solution $(20 \mathrm{ml} ; 0.5 \%[\mathrm{w} / \mathrm{v}])$. Finally, the organic solvent was removed by dialysis in the dialysis bags with a retained molecular weight range of 8000 to 14,000 Da to obtain LDPs. DPs were prepared by the same method.

\section{Characterization of DPs \& LDPs}

The NPs were stained with phosphotungstic acid and attached to carbon-supporting films. The morphology of the NPs was observed by transmission electron microscopy (TEM) using a JEM-1230 microscope (Jeol, Tokyo, Japan). The particle size and $\zeta$-potential of the NPs were characterized by a dynamic light scattering (DLS) instrument (Nicomp 380 ZLS; PSS, CA, USA). The entrapment efficiency (EE) and loading capacity (LC) were determined by ultrafiltration tube centrifugation $(6000 \times g, 20 \mathrm{~min})$ to obtain the free drug in the lower layer and concentrated NPs in the upper layer, the concentrations of which were measured by HPLC. The EE and LC were calculated by the following equations:

$$
\begin{aligned}
& \mathrm{EE}(\%)=\frac{\text { total amount of exenatide }- \text { free exenatide }}{\text { total amount of exenatide }} \times 100 \% \\
& \mathrm{LC}(\%)=\frac{\text { total amount of exenatide }- \text { free exenatide }}{\text { weight of } N P s} \times 100 \%
\end{aligned}
$$

Investigation of mucus aggregation \& penetration

Fresh rat intestinal mucus was used for evaluation in a mucus aggregation test. DPs, LDPs and free fluorescein isothiocyanate (FITC)-exenatide (Shanghai Xinhao Biochemicals [Shanghai, China]; $60 \mu \mathrm{g} / \mathrm{ml}$ ) were dispersed in mucus $(100 \mu \mathrm{l})$, and incubated at $37^{\circ} \mathrm{C}$ under $5 \% \mathrm{CO}_{2} / 95 \% \mathrm{O}_{2}$ on a shaking bed for 1 or $2 \mathrm{~h}$. The supernatants were obtained by centrifugation at $5000 \times g$ for $10 \mathrm{~min}$. DMSO was added to the supernatants to destroy any NPs not adsorbed by the mucin. A CYTATION imaging reader (BioTek, VT, USA) was used to measure the fluorescence intensity.

The mucus permeability of DPs, LDPs and free-FITC-exenatide was examined using a 3D device based on a Transwell ${ }^{\circledR}$ chamber, Costar, ME, USA [30]. Rat intestinal mucus $(100 \mu \mathrm{l})$ was added to each donor chamber, and $1000 \mu \mathrm{l}$ of PBS ( $\mathrm{pH}$ 7.4) was placed in each recipient chamber. After 15 min of equilibrium, $200 \mu \mathrm{l}$ of DPs, LDPs or free-FITC-exenatide $(60 \mu \mathrm{g} / \mathrm{ml})$ was added dropwise to the donor chamber. A $100-\mu \mathrm{l}$ aliquot was removed from each recipient chamber at $1,1.5$ and $2 \mathrm{~h}$, and mixed with $150 \mu \mathrm{l}$ of DMSO to destroy the NPs. At the same time, $100 \mu \mathrm{l}$ of fresh PBS was supplemented to the recipient chamber. The concentrations of the samples at the 
different time points were measured by a CYTATION imaging reader. The apparent permeability coefficient $\left(\mathrm{P}_{\text {app }}\right)$ value was calculated as follows:

$$
\mathrm{P}_{\text {app }}=\frac{\mathrm{dQ}}{\mathrm{dt}} \times \frac{1}{A \times \mathrm{C} 0}
$$

where $\mathrm{dQ} / \mathrm{dt}$ is the flux of FITC from the donor side to the recipient side; $\mathrm{A}$ is the filter membrane area $\left(\mathrm{cm}^{2}\right)$; and $\mathrm{C}_{0}$ is the initial concentration of FITC in the donor chamber.

\section{Cell experiments \\ Cellular uptake experiment}

Glass slides were washed with sterilized water and laid flat in the wells of a 24-well cell plate, followed by addition of $3 \times 10^{4}$ Caco- 2 cells and incubation for $48 \mathrm{~h}$. The cells were further incubated with coumarin-6-labeled DPs or LDPs $(100 \mathrm{ng} / \mathrm{ml})$ for $0.5,1,2$ or $4 \mathrm{~h}$. The extracellular NPs were washed with PBS, and the cells were immobilized with $4 \%$ paraformaldehyde. The paraformaldehyde was washed out with PBS, and the nuclei were stained with Hoechst 33342 solution. The glass slides were removed and observed with a high-resolution live cell imaging system.

Caco- 2 cells were inoculated in a six-well plate at $3 \times 10^{5}$ cells/well and incubated for $48 \mathrm{~h}$. The culture medium was discarded and the cells incubated with coumarin-6-labeled DPs and LDPs ( $4 \mathrm{ng} / \mathrm{ml})$ for $0.5,1,1.5$ or $3 \mathrm{~h}$. The NP solutions were discarded, and the cells were digested with trypsin and washed with PBS. The fluorescence intensity was measured by flow cytometry as described [31].

\section{Cellular uptake mechanism experiment}

To evaluate the mechanism for the interactions with DPs and LDPs, Caco-2 cells were inoculated into six-well plates at $3 \times 10^{5}$ cells/well and incubated for $48 \mathrm{~h}$. After removal of the cell culture medium, $2 \mathrm{ml}$ of formalin $(20 \mu \mathrm{mol} / \mathrm{l})$, chlorpromazine $(30 \mu \mathrm{mol} / \mathrm{l})$ or $\mathrm{M}-\beta-\mathrm{CD}(2.5 \mathrm{mM})$ was added and the cells were incubated for $0.5 \mathrm{~h}$. The inhibitors were discarded and the cells were incubated with coumarin-6-labeled DPs or LDPs ( $4 \mathrm{ng} / \mathrm{ml})$ for $1 \mathrm{~h}$. The NP solutions were discarded, and the cells were digested with trypsin and washed with PBS. The fluorescence intensity was measured by flow cytometry as described [13,32].

\section{In vitro transepithelial transport experiments}

A Caco-2/HT29-MTX co-culture model was used to simulate transmembrane transport through a monolayer. Caco-2 and HT29-MTX cells (8:1) were seeded in transwell plates at $2 \times 10^{5} /$ well and incubated at $37^{\circ} \mathrm{C}$ under $5 \% \mathrm{CO}_{2}$ for 21 days until the cell resistance was $300-400 \Omega / \mathrm{cm}^{2}$, as appropriate in experiments.

Before experiments, the culture medium was replaced with fresh prewarmed Hank's buffered salt solution (HBSS) and the cells were incubated at $37^{\circ} \mathrm{C}$ for $30 \mathrm{~min}$. Next, $500 \mu \mathrm{l}$ of FITC-exenatide-DPs, FITC-exenatide-LDPs, FITC-exenatide-LDPs + free LMWP or FITC-exenatide-DPs + free LMWP was added to the upper chambers at $60 \mu \mathrm{g} / \mathrm{ml}$ FITC-exenatide and $1 \mathrm{mg} / \mathrm{ml}$ LMWP. Fresh prewarmed HBSS was added to the lower chambers and incubated for $4 \mathrm{~h}$. Samples $(500 \mu \mathrm{l})$ were collected from the basolateral chambers at predetermined times $(0.5$, 1, 2, 3 and $4 \mathrm{~h}$ ) and replaced with equal volumes of fresh HBSS. The amount of transported FITC-exenatide was determined by a microplate reader, and the cumulative transport of FITC-exenatide was calculated [31]. Each experiment was performed in triplicate. The $\mathrm{P}_{\text {app }}$ was calculated by the following equation:

$$
\mathrm{B}_{\mathrm{R}}(\%)=\operatorname{AUC}(\text { p.o }) \times \operatorname{dose}(\text { s.c. }) / \operatorname{AUC}(\text { s.c. }) \times \operatorname{dose}(\text { p.o. }) \%
$$

where $\mathrm{Q}(\mathrm{t})$ is the cumulative amount of exenatide transported to the basolateral side $(\mu \mathrm{g})$; $\mathrm{A}$ is the area of the cell monolayer $\left(\mathrm{cm}^{2}\right) ; \mathrm{C}_{0}$ is the initial concentration in the upper chamber; and $\mathrm{dQ}(\mathrm{t}) / \mathrm{dt}$ is the slope of the linear relationship between the cumulative amount of exenatide transported $(\mu \mathrm{g})$ versus time $(s)$.

\section{Distribution of DPs \& LDPs in vivo}

1,1'-dioctadecyl-3,3,3',3'-tetramethyl indotricarbocyanine Iodide (DiR)-labeled DPs or LDPs were prepared by single emulsification method. The $10 \mathrm{mg}$ PLGA was completely dissolved in $0.3 \mathrm{ml}$ DMSO, and $0.1 \mathrm{ml}(100 \mathrm{mg} / \mathrm{ml}$ 
DiR acetone solution) and $0.6 \mathrm{ml}$ dichloromethane were added, which was mixed as oil phase. The oil phase was transferred to $5 \mathrm{ml}$ polyvinyl alcohol (PVA) solution (w/v 1\%) and the emulsion was obtained by $300 \mathrm{~W}$ ultrasound (ultrasonic 30-times, $10 \mathrm{~s}$ bursts, $5 \mathrm{~s}$ intervals). The composite emulsion was dispersed into polyvinyl alcohol solution $(20 \mathrm{ml} ; 0.5 \%$ [w/v]). Finally, the organic solvent was removed by dialysis in the dialysis bags with a retained molecular weight range of 8000-14,000 Da to obtain DiR-labeled DPs or LDPs.

All animal experiments were approved by the Experimental Animal Ethics Committee of the School of Pharmacy, Yantai University. The mice were divided into two groups with eight rats per group. One group was given DiRlabeled DPs, and the other group was given DiR-labeled LDPs $(5 \mu \mathrm{g} / 10 \mathrm{~g})$. The heart, liver, spleen, lung and kidney were isolated from six rats at 1, 2, 4, 6, 12 and $24 \mathrm{~h}$ after oral administration, and the tissue distributions of DiR were observed with an in vivo imaging system by using the In-Vivo Imaging System FX Pro (Carestream, NY, USA) with an excitation of $720 \mathrm{~nm}$ and an emission of $790 \mathrm{~nm}$. The images were analyzed using the Carestream Image Suite Software. The remaining two rats in each group were anesthetized with chloral hydrate, and the distribution of DiR was observed at $0.5,1,2,3,4,6,8,10,12$ and $24 \mathrm{~h}$, respectively, as described previously [14].

\section{Permeability of the intestinal epithelium}

Male Sprague-Dawley rats (weight: 220-250 g) were divided into two groups. The rats had free access to deionized water after fasting for $12 \mathrm{~h}$ before the experiment. Nile red-labeled DPs and LDPs $(100 \mu \mathrm{g} / \mathrm{kg})$ were administered intragastrically for $150 \mathrm{~min}$ and the rats were euthanized. Approximately $2 \mathrm{~cm}$ of the duodenum, jejunum, ileum, cecum, colon and rectum was taken and washed with normal saline. The tissue was fixed, dehydrated, embedded with an optimum cutting temperature compound, frozen and sectioned. The section was observed under a confocal laser scanning microscope (FluoView FV1000; Olympus, Tokyo, Japan).

In another experiment, male Sprague-Dawley rats weighing 220-250 g were fasted for $12 \mathrm{~h}$ with free access to deionized water. Under anesthesia, approximately $2 \mathrm{~cm}$ of the duodenum, jejunum, ileum and colon was removed. The intestinal contents were washed out with normal saline and the tissue was ligated at both ends. Next, $0.1 \mathrm{ml}$ of DP or LDP solution was injected into four intestinal rings per group and the tissues were incubated in PBS $(\mathrm{pH}$ 7.4; $10 \mathrm{ml}$ ) at $37^{\circ} \mathrm{C}$ under $95 \% \mathrm{O}_{2}$ and $5 \% \mathrm{CO}_{2}$. After incubation for $150 \mathrm{~min}, 50-\mu \mathrm{l}$ samples were removed from each loop and DMSO $(50 \mu \mathrm{l})$ was used for demulsification. The fluorescence intensity of FITC in the samples was measured. The two ends of the loop were then opened and cleaned with PBS. The area of each ring was measured and the relative intestinal transport per unit area was calculated [32].

\section{In vivo pharmacokinetic study}

All animal experiments were carried out with approval from the Experimental Animal Ethics Committee of the School of Pharmacy, Yantai University. Male Sprague-Dawley rats were randomly divided into five groups. The rats were fasted $12 \mathrm{~h}$ before experiments, but had free access to drinking water. One group was subcutaneously administered exenatide solution $(10 \mu \mathrm{g} / \mathrm{kg})$, and the other three groups were orally administered exenatide- $\mathrm{Zn}^{2+}$ solution, exenatide- $\mathrm{Zn}^{2+}$-DPs and exenatide- $\mathrm{Zn}^{2+}$-LDPs $(100 \mu \mathrm{g} / \mathrm{kg})$. Blood samples were taken from the retinal veins at $0,5,15,30$ and $45 \mathrm{~min}$ and at 1, 2, 4, 6, 8, 10, 12 and $24 \mathrm{~h}$. The Exendin-4 ELISA Kit was used to determine the serum levels of exenatide [31]. The relative bioavailability of NPs was calculated by the following formula:

$$
\mathrm{B}_{\mathrm{R}}(\%)=\operatorname{AUC}(\text { p.o }) \times \operatorname{dose}(\text { s.c. }) / \operatorname{AUC}(\text { s.c. }) \times \operatorname{dose}(\text { p.o. }) \%
$$

where AUC is the area under the curve, p.o. is per oral, s.c. is subcutaneous.

Pharmacodynamic study

Single administration

In this study, $\mathrm{db} / \mathrm{db}$ mice were used as an animal model of Type 2 diabetes. Male 6-week-old $\mathrm{db} / \mathrm{db}$ mice were randomly divided into five groups, and treated with oral administration of exenatide- $\mathrm{Zn}^{2+}$ solution $(100 \mathrm{\mu g} / \mathrm{kg}$ exenatide), exenatide- $\mathrm{Zn}^{2+}$-DPs (100 $\mu \mathrm{g} / \mathrm{kg}$ exenatide), exenatide- $Z \mathrm{n}^{2+}$-LDPs (100 $\mu \mathrm{g} / \mathrm{kg}$ exenatide), or saline, or a subcutaneous injection of exenatide solution $(10 \mu \mathrm{g} / \mathrm{kg})$. Blood samples were collected from the caudal vein at 0 and $30 \mathrm{~min}$, and at 1, 2, 4, 6, 8, 10, 12 and $24 \mathrm{~h}$, and blood glucose levels were measured with a blood glucose meter (ACCU-CHEK ${ }^{\circledR}$; Integra, Germany) as described [14,31]. 


\begin{tabular}{|c|c|c|c|c|c|}
\hline DPs & $95.4 \pm 6.1$ & $0.142 \pm 0.038$ & $0.56 \pm 0.017$ & / & l \\
\hline LDPs & $123.1 \pm 5.4$ & $0.175 \pm 0.053$ & $0.49 \pm 0.022$ & / & / \\
\hline Exenatide- $\mathrm{Zn}^{2+}-\mathrm{DPs}$ & $102.4 \pm 7.1$ & $0.181 \pm 0.042$ & $0.38 \pm 0.025$ & $75.73 \pm 2.07$ & $5.57 \pm 0.55$ \\
\hline
\end{tabular}

DP: Dextran-poly(lactic-co-glycolic acid)-nanoparticle; EE: Entrapment efficiency; LC: Loading capacity; LDP: Low molecular weight protamine-dextran-poly(lactic-co-glycolic acid)nanoparticle; PDI: Polydispersity index.

\section{Multiple administrations}

6-week-old male $\mathrm{db} / \mathrm{db}$ mice were randomly divided into five groups. One group received subcutaneous administration twice a day for 5 days and the other four groups received oral administration once a day for 5 days with the solutions described above. Blood samples were collected from the tail vein at $0,1,2,3,4$ and 5 days $(24 \mathrm{~h}$ after administration) for determination of blood glucose levels as described [31]. The dry or wet conditions of the cages in all groups were checked daily to evaluate the amounts of urine produced by the mice.

\section{Statistical analysis}

Data are expressed as mean \pm standard deviation. A two-tailed Student's t-test was used to determine the significance of differences between groups. Values of $\mathrm{p}<0.05$ were considered significant, and values of $\mathrm{p}<0.01$ were considered highly significant.

\section{Results}

\section{Characterization of DPs \& LDPs}

Table 1 summarizes the characteristics of the blank and drug-loaded NPs. Compared with the blank batch $(95.4 \pm 6.1 \mathrm{~nm} ; 0.56 \pm 0.017 \mathrm{mV})$, the drug-loaded DPs had larger particle size $(102.4 \pm 7.1 \mathrm{~nm})$ and lower $\zeta$ potential $(0.38 \pm 0.027 \mathrm{mV})$, arising from the encapsulation of the negatively charge exenatide resulting in a decrease in the overall potential of the NPs. In addition, both blank LDPs and exenatide- $\mathrm{Zn}^{2+}$-LDPs had lower $\zeta$-potential and larger particle size than DPs. The particle size of exenatide- $\mathrm{Zn}^{2+}{ }_{-} \mathrm{DPs}$ was $102.4 \pm 7.1 \mathrm{~nm}$ and the $\zeta$-potential was $0.38 \pm 0.025 \mathrm{mV}$, while the particle size of exenatide- $\mathrm{Zn}^{2+}$-LDPs was $141.1 \pm 6.9 \mathrm{~nm}$ (Figure $2 \mathrm{~A}$ ) and the $\zeta$-potential was $0.16 \pm 0.027 \mathrm{mV}$ (Figure 2B). This would be caused by the existence of the negatively charged LMWP. The DPs and LDPs were uniformly spherical in TEM images (Figure 2C). The EE of DPs was $75.73 \pm 2.07 \%$ and the LC was $5.57 \pm 0.55 \%$, while the EE of LDPs was $68.94 \pm 2.84 \%$ and the LC was $4.62 \pm 0.73 \%$.

\section{Aggregation \& permeability of mucus}

As shown in Figure 3A, the relative retention of free FITC-exenatide was about $80 \%$ at $1 \mathrm{~h}$, while that of DPs and LDPs was about $90 \%$. This may be caused by the strong negative charge of free FITC-exenatide. The existence of LMWP did not significantly increase the interaction between LDPs and mucus.

As shown in Figure 3B, the amount of mucus penetration of NPs clearly increased over time. The penetration rate of LDPs was 1.5-times higher than that of DPs and 2-times higher than that of free FITC-exenatide. This was because LDPs were electrically neutral as a whole, and the small amount of LMWP attached to the outer layer of LDPs had a weak negative electronegativity.

\section{Cell experiments}

Cellular uptake experiment

After treatment with coumarin-6-labeled DPs or LDPs, the images of Caco-2 cells were captured by high-resolution live cell image system, as shown in Figure 4A. In the images, nuclei labeled with Hoechst 33342 appeared blue and coumarin-6-labeled NPs appeared green, revealing that NPs were present in the cytoplasm rather than the nucleus. The fluorescence intensity increased over time, indicating that the uptake of NPs was time dependent. 
(A) $\mathrm{Re}$

Intens-wt gaussian distribution

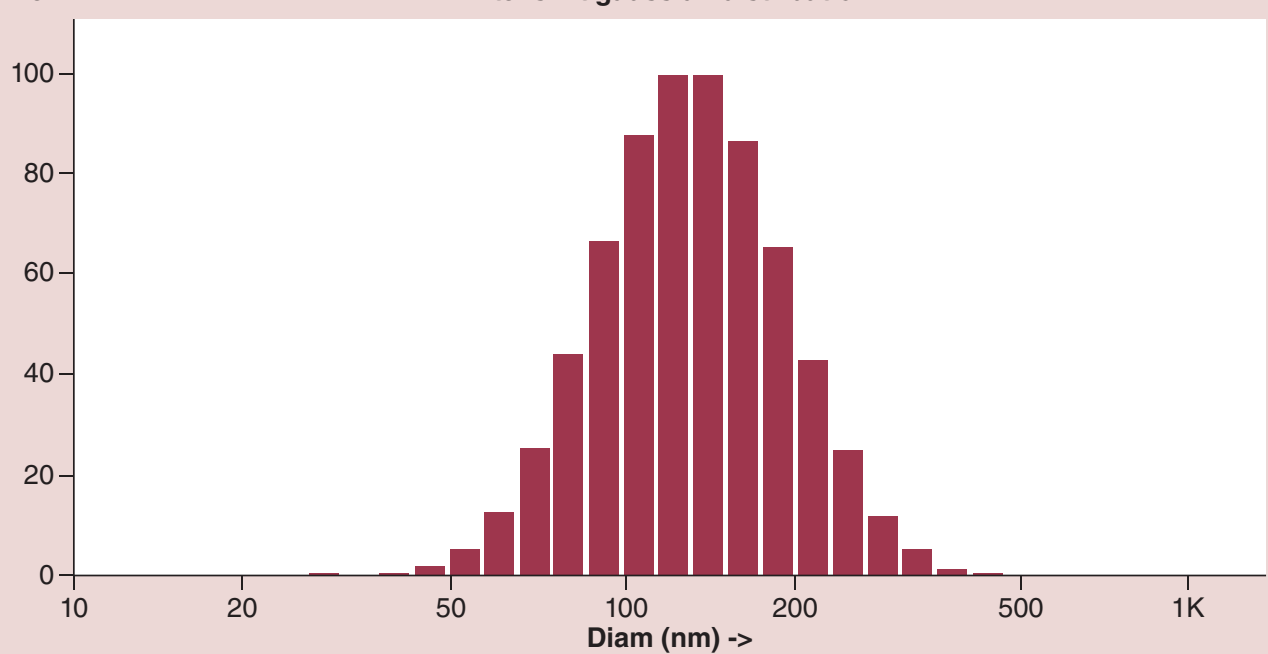

(B) Run_sample

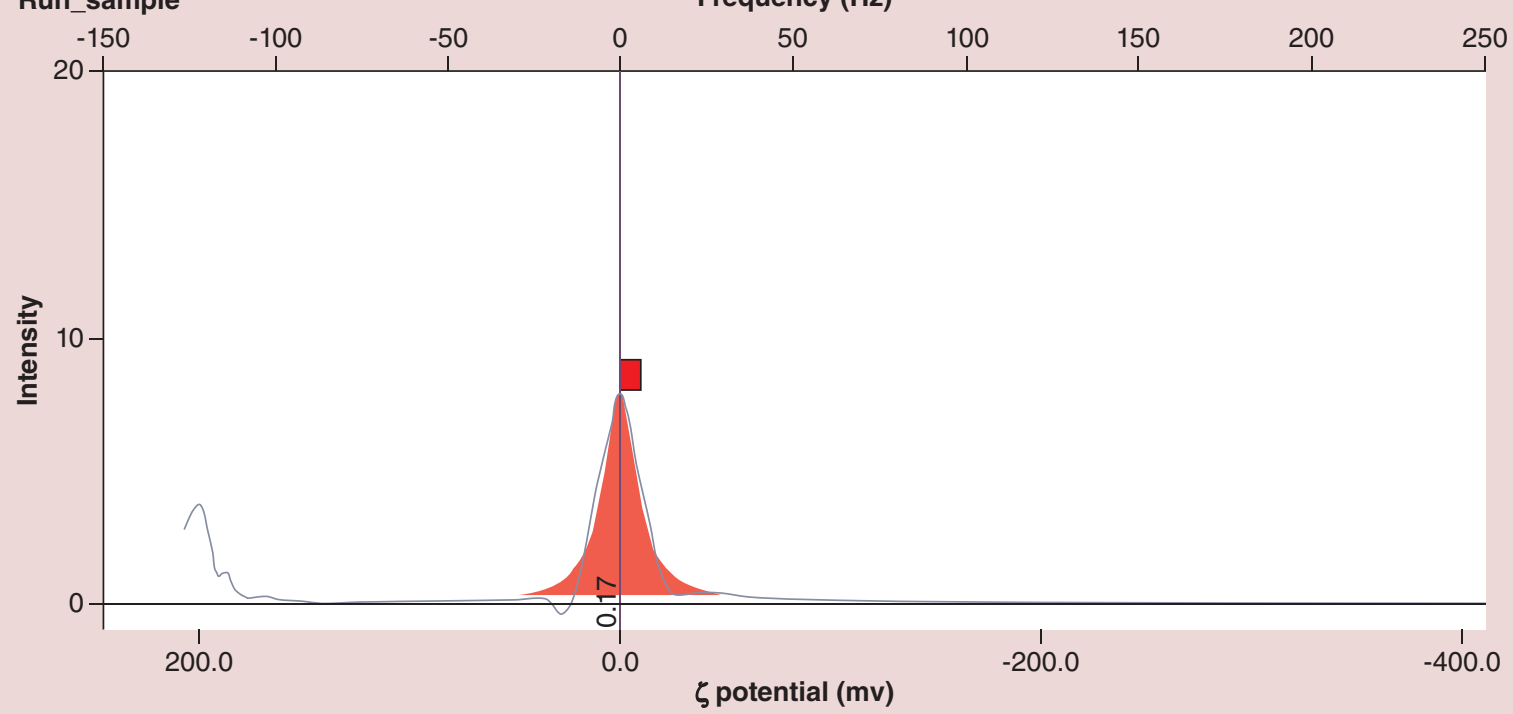

(c)

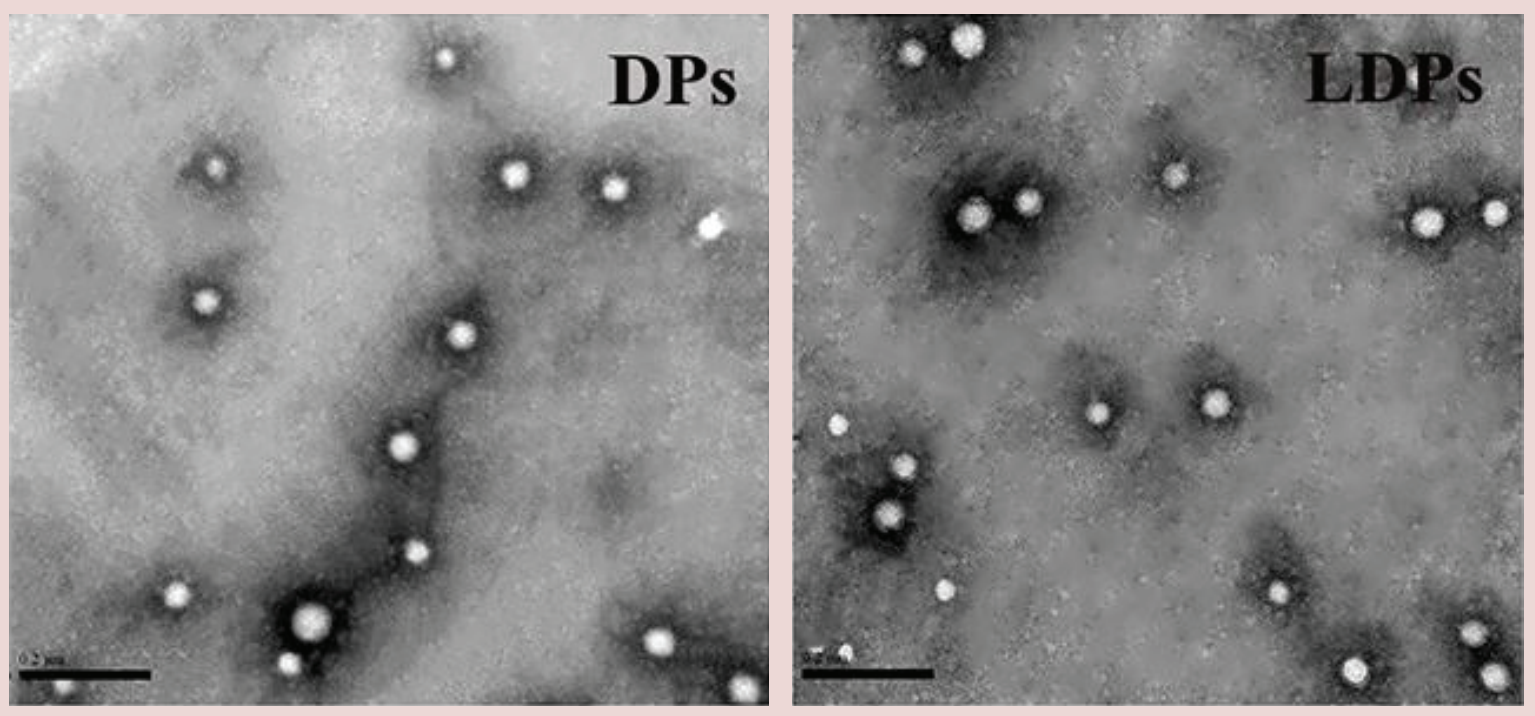

Figure 2. Characterization of nanoparticles in vitro. (A) Particle size distribution of exenatide-Zn ${ }^{2+}-$ LDPs measured by the dynamic light scattering method. (B) $\zeta$-potential diagram of exenatide-Zn ${ }^{2+}$-LDPs. (C) Transmission electron microscopy images of dextran-poly(lactic-co-glycolic acid)-nanoparticles and LDPs. Scale bars: $200 \mathrm{~nm}$.

LDP: Low molecular weight protamine-dextran-poly(lactic-co-glycolic acid)-nanoparticle. 
(A)

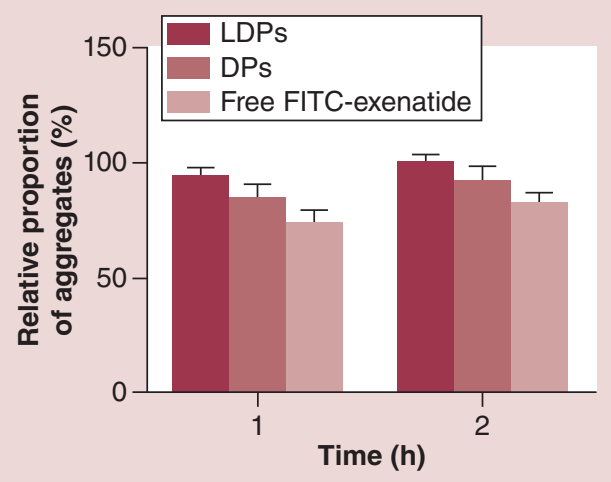

(B)

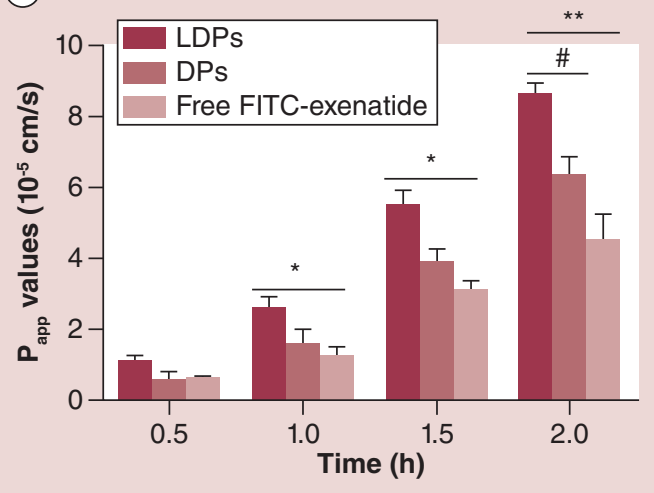

Figure 3. Effect of mucus on nanoparticles. (A) Percentages of mucin particle aggregation in rat intestinal mucus for DPs and low molecular weight protamine-dextran-poly(lactic-co-glycolic acid)-nanoparticles at $37^{\circ} \mathrm{C}$. (B) Apparent permeability coefficient values of DPs and low molecular weight protamine-dextran-poly(lactic-co-glycolic acid)-nanoparticles during incubation with mucus for $2 \mathrm{~h}$ in a mucus penetration study. Data represent means \pm standard deviation $(n=3)$. ${ }^{*} p<0.05,{ }^{*} p<0.01$ versus FITC-exenatide groups; \#p $<0.05$, versus DPs groups. DP: Dextran-poly(lactic-co-glycolic acid)-nanoparticle; LDP: Low molecular weight protamine-dextran-poly(lactic-co-glycolic acid)-nanoparticle.

Table 2. Nanoparticle permeability across cell monolayers.

\begin{tabular}{|ll|}
\hline Formulation & Permeability $\left(\mathbf{P}_{\text {app }}\right)(\mathbf{c m} / \mathbf{s}) \times 10^{-8}$ \\
\hline DPs & $13.55 \pm 0.32$ \\
\hline LDPs & $19.32 \pm 0.17$ \\
\hline Free LMWP+DPs & $12.46 \pm 0.41$ \\
\hline Free LMWP+LDPs & $15.21 \pm 0.37$ \\
\hline
\end{tabular}

DP: Dextran-poly(lactic-co-glycolic acid)-nanoparticle; LDP: Low molecular weight protamine-dextran-poly(lactic-co-glycolic acid)-nanoparticle; LMWP: Low molecular weight protamine.

Quantitative analyses of the cellular internalization of coumarin-6-labeled DPs and LDPs were performed by flow cytometry (Figure 4B). LDPs exhibited significantly higher counts $(\mathrm{p}<0.05)$ than DPs at all time points, indicating that LMWP promoted the uptake of LDPs. The uptake amount of LDPs was twofold higher $(\mathrm{p}<0.05)$ than that of unmodified DPs at $0.5 \mathrm{~h}$. At $3 \mathrm{~h}$, the fluorescence intensity in the LDP group was still stronger than that in the DP group.

\section{Cellular uptake mechanism experiment}

The relative amounts of uptake compared with the untreated control group are shown in Figure 6C. The uptake amounts of both DPs and LDPs were reduced in the presence of M- $\beta-C D$, indicating the involvement of an energydependent active transport process. After incubation with chlorpromazine, the uptake of LDPs was decreased by about $25 \%$, showing that clathrin was involved in the transport of LDPs. The uptake of LDPs was decreased by about $40 \%$ in formalin-treated cells, indicating that caveolae were involved in LDP transport.

\section{In vitro transepithelial transport studies}

The permeability values of various formulations are given in Table 2 . The $P_{\text {app }}$ of the LDPs was $19.32 \times 10^{-8} \mathrm{~cm} / \mathrm{s}$, the $\mathrm{P}_{\text {app }}$ of DPs was $13.55 \times 10^{-8} \mathrm{~cm} / \mathrm{s}$, and the $\mathrm{P}_{\text {app }}$ of LDPs + LMWP was $15.21 \times 10^{-8} \mathrm{~cm} / \mathrm{s}$. As shown in Figure 4D, the amount of FITC-exenatide in the lower chamber increased over time, indicating that the transmembrane transport of FITC-exenatide was time dependent. After $2 \mathrm{~h}$, the FITC-exenatide content in the lower chamber in the LDP groups was 1.5-times higher than that in the DPs groups. The LDP groups had higher capacity than the free LMWP + LDP groups at all time points. 
(A)

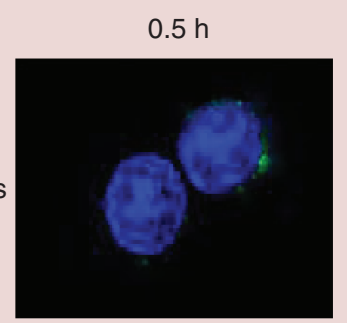

LDPs

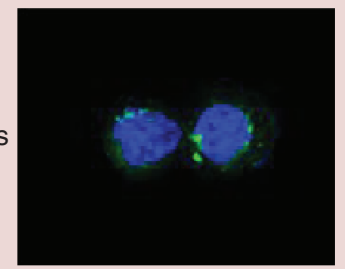

(B)

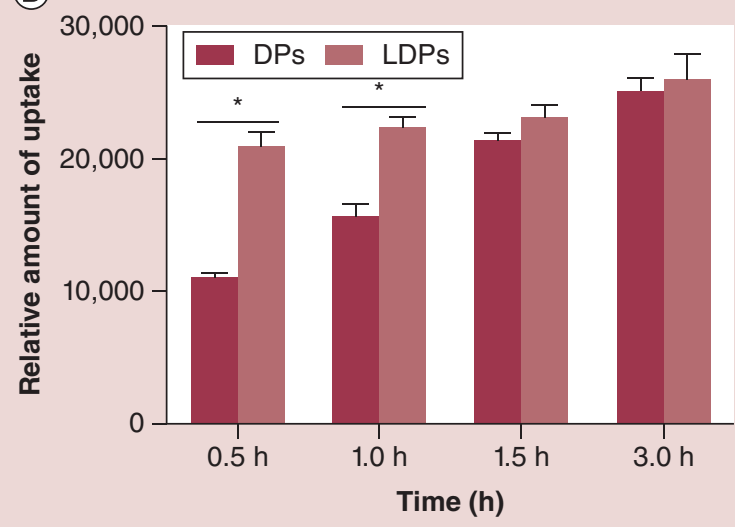

$2.0 \mathrm{~h}$
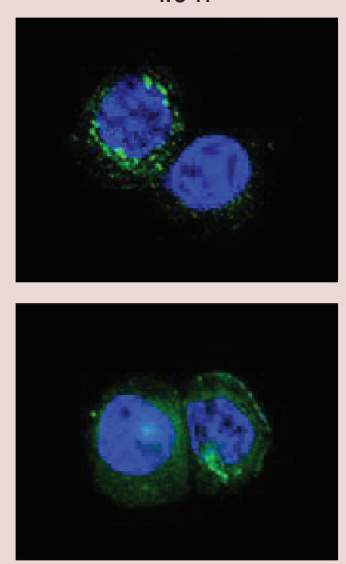
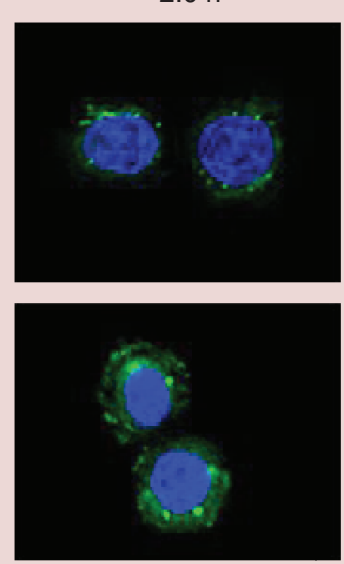

$4.0 \mathrm{~h}$
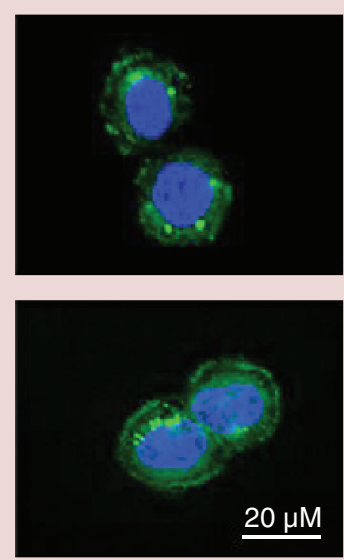

(C)

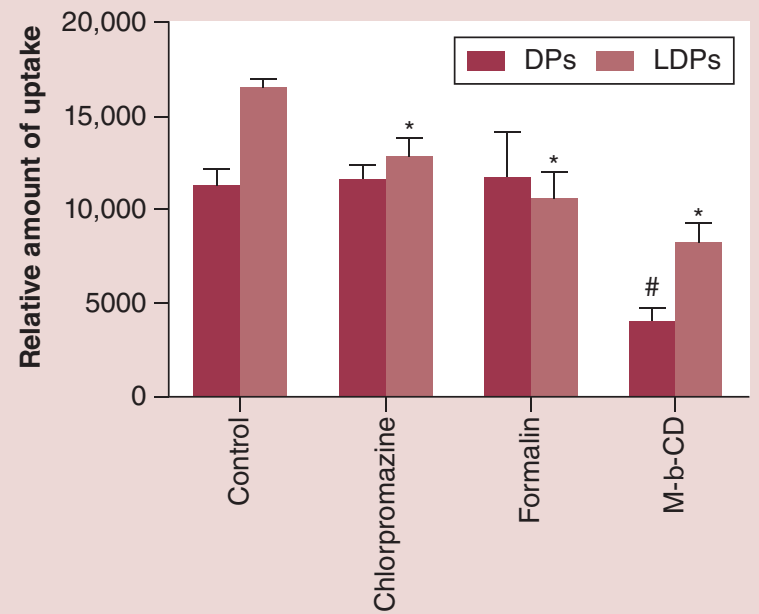

(D)

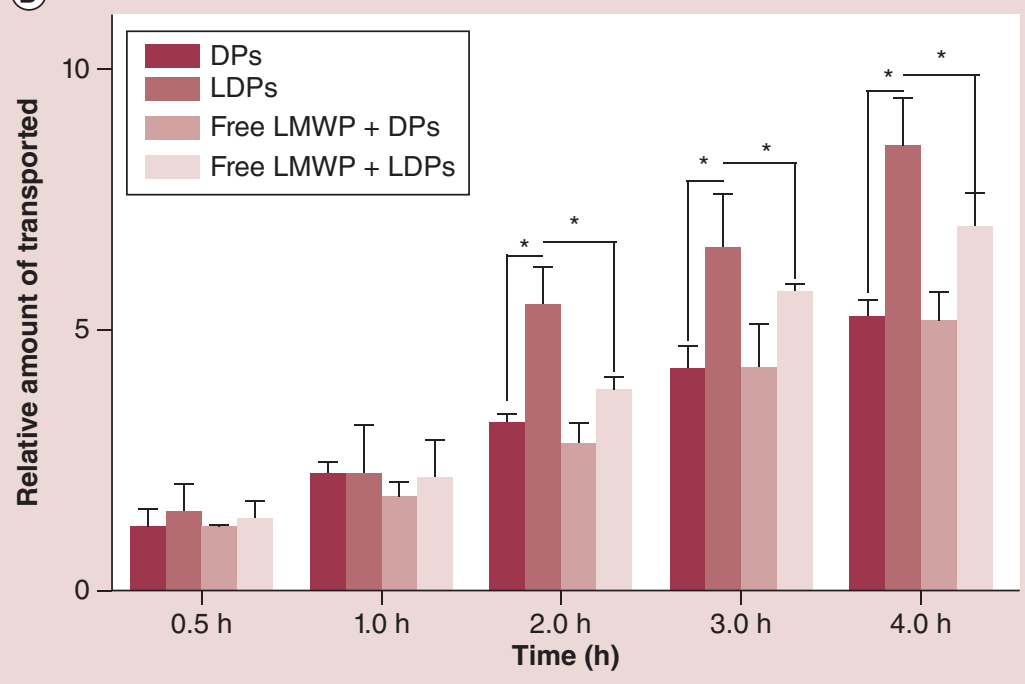

Figure 4. In vitro cell uptake. (A) High-resolution live cell imaging system images of Caco-2 cells after incubation with LDPs or DPs. The green signals indicate internalized nanoparticles. The blue signals indicate nuclei. (B) Relative fluorescence intensity of coumarin-6 in Caco- 2 cells. Data represent means \pm SD $(n=3)$. ${ }^{*} p<0.05$, LDPs versus DPs. (C) Relative amounts of uptake of LDPs and DPs by Caco- 2 cells in the presence of different endocytosis inhibitors. Data represent means \pm SD $(n=3)$. ${ }^{*} p<0.05$; ${ }^{\#} p<0.05$, versus control. (D) Cumulative amounts of FITC-exenatide transported across the cell monolayers. Data represent $m e a n s \pm S D(n=3)$.

DP: Dextran-poly(lactic-co-glycolic acid)-nanoparticle; LDP: Low molecular weight protamine-dextran-poly(lactic-co-glycolic acid)-nanoparticle; SD: Standard deviation. 

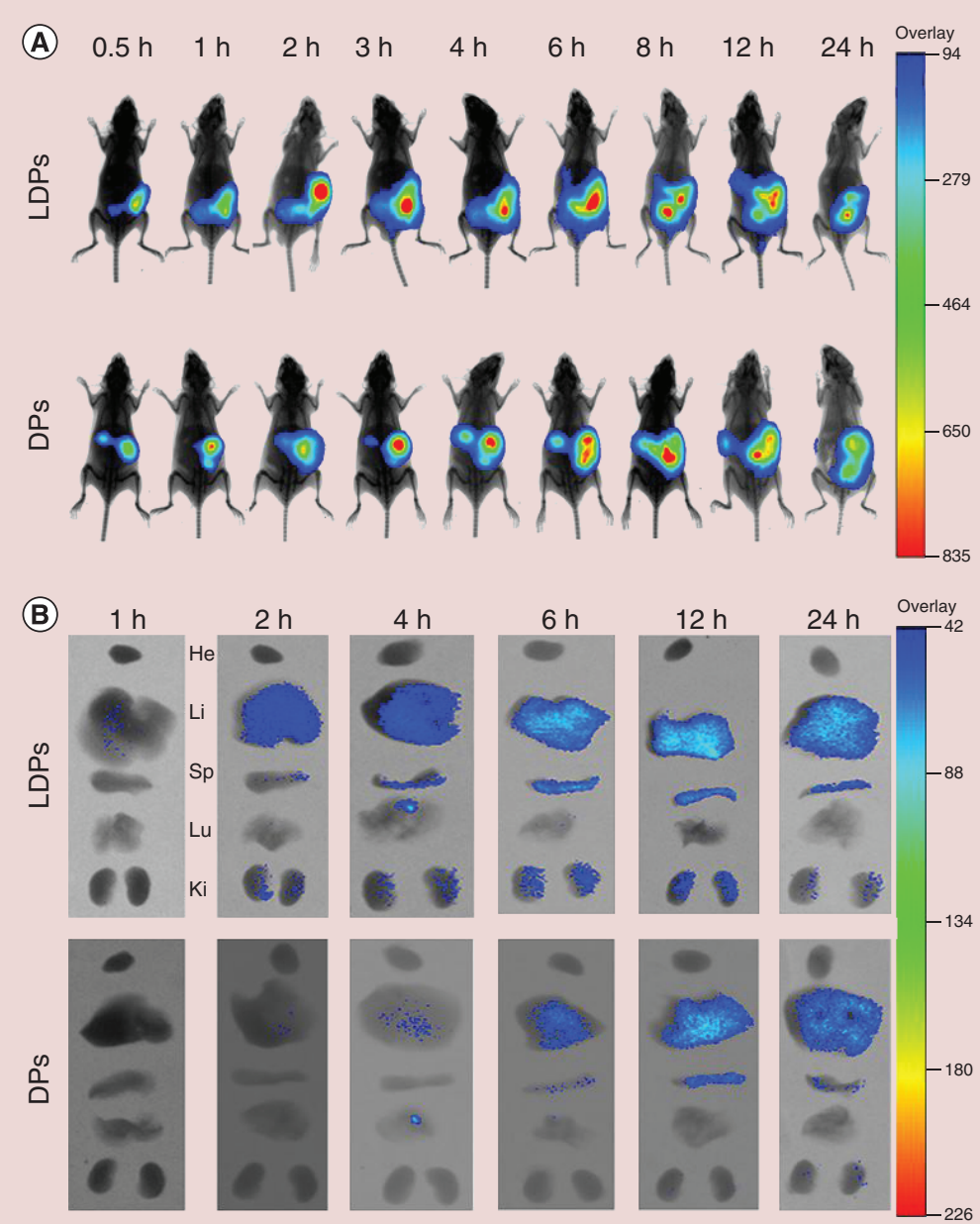

Figure 5. Distribution of nanoparticles in vivo. (A) In vivo distribution of DiR in rats monitored by in vivo imaging after oral administration of dextran-poly(lactic-co-glycolic acid)-nanoparticles or low molecular weight protamine-dextran-poly(lactic-co-glycolic acid)-nanoparticles. (B) Fluorescence images of anatomical organs. DP: Dextran-poly(lactic-co-glycolic acid)-nanoparticle; He: Heart; LDP: Low molecular weight protamine-dextran-poly(lactic-co-glycolic acid)-nanoparticle; Li: Liver; Sp: Spleen; Lu: Lung; Ki: Kidney.

\section{Distribution of DPs \& LDPs in vivo}

The observations in live mice at different time points are shown in Figure 5A. It can be seen that the fluorescence area increased over time. Compared with the DP group, the fluorescence area in the LDP group expanded more rapidly. Figure 5B reveals the presence of weak fluorescence in the liver of the LDP group at $1 \mathrm{~h}$, but no sign of fluorescence in the DP group. Strong fluorescence was observed in the liver of the LDP group at $2 \mathrm{~h}$, but there was still no sign of fluorescence in the DP group. At later time points, the LDP group continued to show strong fluorescence in the liver, spleen and kidney. In the DP group, the liver began to show strong fluorescence intensity at $6 \mathrm{~h}$. However, at 12 and $24 \mathrm{~h}$, the fluorescence intensity in the DP group remained weaker than that in the LDP group. These findings indicated that the absorption of LDPs was faster and more widespread than that of DPs [32].

\section{Permeability of the intestinal epithelium}

The absorption of LDPs and DPs in the small intestine was higher than that in the large intestine (Figure 6A \& B). However, the fluorescence intensity of LDPs was stronger than that of DPs in both the large and small intestines. In the large intestine, the fluorescence intensity in the colon was stronger than that in the cecum and rectum. 


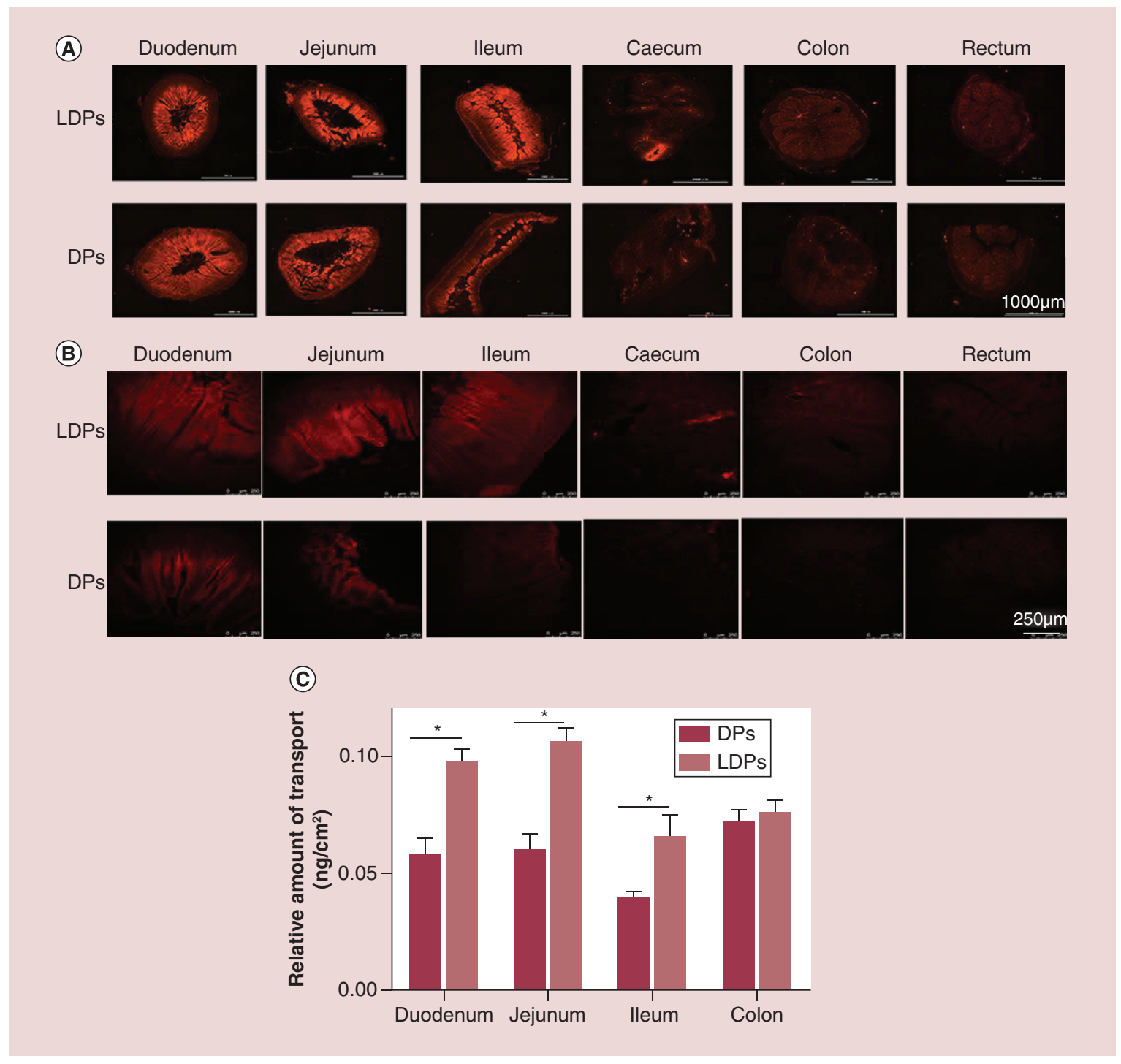

Figure 6. Absorption of nanoparticles in different intestinal segments. (A) Cross-sectional images of different parts of the intestine observed with the CYTATION imaging instrument. (B) Villus images of different intestinal segments observed with a confocal laser scanning microscope. (C) Volumes per unit area of low molecular weight protamine-dextran-poly(lactic-co-glycolic acid)-nanoparticles and dextran-poly(lactic-co-glycolic acid)-nanoparticles in the intestinal ligature test in vitro. Data represent means \pm standard deviation $(n=3)$. ${ }^{*} p<0.05$, low molecular weight protamine-dextran-poly(lactic-co-glycolic acid)-nanoparticles versus dextran-poly(lactic-co-glycolic acid)-nanoparticles.

DP: Dextran-poly(lactic-co-glycolic acid)-nanoparticle; LDP: Low molecular weight protamine-dextran-poly(lactic-co-glycolic acid)-nanoparticle.

As shown in Figure 6C, the transport capacity of LDPs in the duodenum, jejunum and ileum was about 1.5-times higher than that of DPs. There was no difference in transport volume between LDPs and DPs in the colon. The results for LDPs and DPs were similar in the colon.

\section{In vivo pharmacokinetic studies}

The plasma exenatide concentration time profiles and the related pharmacokinetic parameters are shown in Figure 7 and Table 3, respectively. The bioavailability in the LDP groups was $8.4 \%$, and significantly higher than the DP groups $(5.3 \%)$ and solution groups $(2.7 \%)$. In both the LDP and DP groups, the highest serum exenatide level existed at $8 \mathrm{~h}$, which was $7.25 \mathrm{~h}$ later than that in the subcutaneous injection groups. In contrast, the maximal 


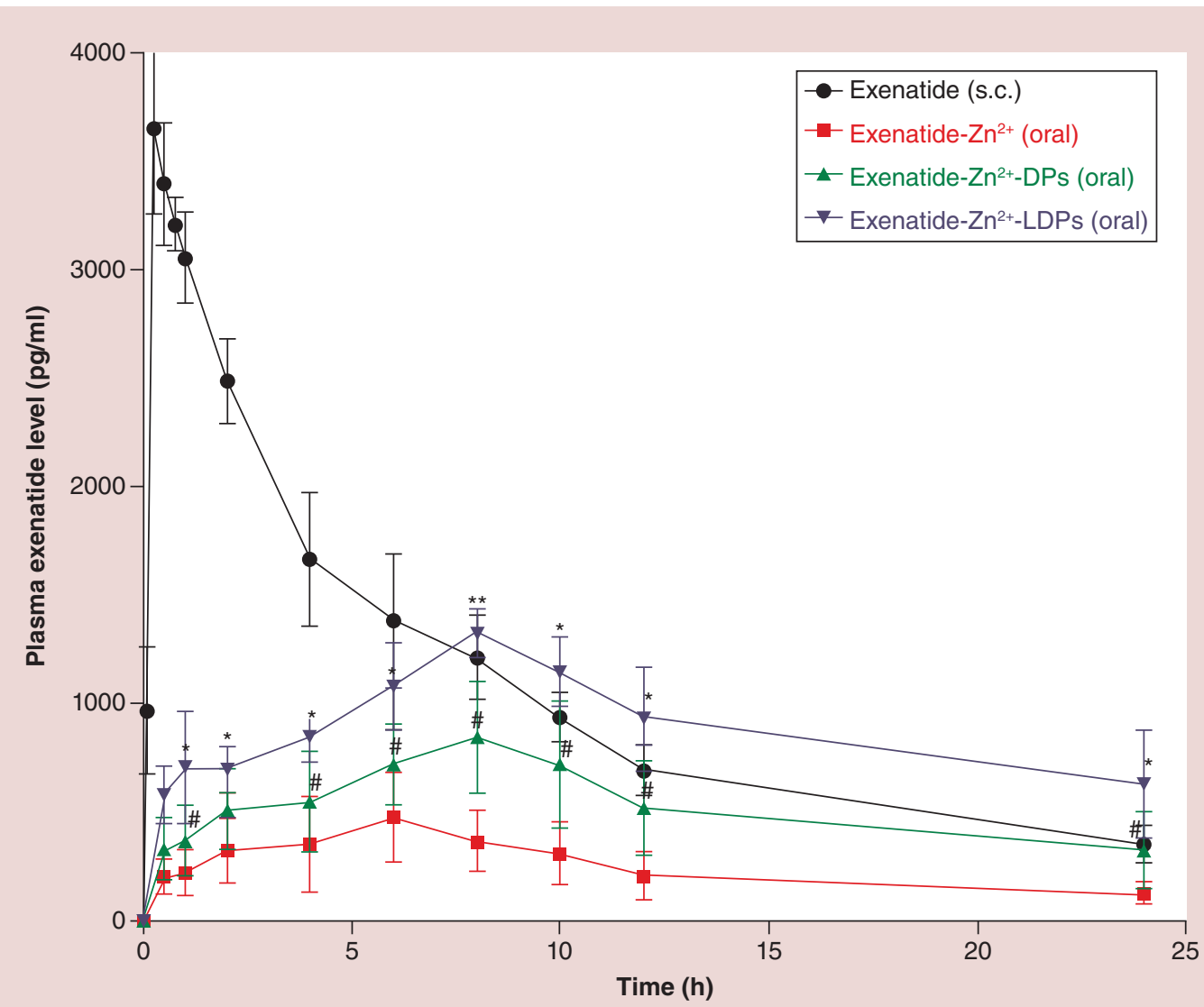

Figure 7. Plasma exenatide level versus time profles of diabetic rats following the administration of different exenatide formulations. Plasma exenatide levels in Sprague-Dawley rats following oral administration of exenatide- $Z \mathrm{n}^{2+}$ solution $(100 \mu \mathrm{g} / \mathrm{kg})$, exenatide- $Z \mathrm{n}^{2+}$-dextran-poly(lactic-co-glycolic acid)-nanoparticles $(100 \mu \mathrm{g} / \mathrm{kg})$ or exenatide-Zn $\mathrm{n}^{2+}$-low molecular weight protamine-dextran-poly(lactic-co-glycolic acid)-nanoparticles $(100 \mu \mathrm{g} / \mathrm{kg})$, or subcutaneous administration of exenatide solution $(10 \mu \mathrm{g} / \mathrm{kg})$ as a positive control. Data represent means \pm standard deviation $(n=5)$. ${ }^{*} p<0.05,{ }^{* *} p<0.01$, versus the oral exenatide- $Z n^{2+}$ solution group. $\# p<0.05$, versus the exenatide-Zn ${ }^{2+}$-dextran-poly(lactic-co-glycolic acid)-nanoparticles group.

Table 3. Pharmacokinetic parameters of exenatide following administration of different exenatide formulations.

\begin{tabular}{|c|c|c|c|c|}
\hline Pharmacological parameters & Exenatide- $-\mathrm{n}^{2+}$ solution (i.g.) & Exenatide-Zn ${ }^{2+}$ DPs (i.g.) & Exenatide-Zn ${ }^{2+}-$ LDPs (i.g.) & Exenatide solution (s.c.) \\
\hline Dose $(\mu \mathrm{g} / \mathrm{ml})$ & 100 & 100 & 100 & 10 \\
\hline $\mathrm{C}_{\max }(\mathrm{pg} / \mathrm{ml})$ & $532.14 \pm 153.25$ & $892.898 \pm 101.79$ & $1353.678 \pm 137.51$ & $3627.86 \pm 471.28$ \\
\hline$T_{\max }(h)$ & 6 & 8 & 8 & 0.25 \\
\hline $\mathrm{AUC}(\mathrm{pgh} / \mathrm{ml})$ & $6697.139 \pm 131.62$ & $13434.544 \pm 1673.21$ & $21203.298 \pm 1108.42$ & $25157.322 \pm 2135.60$ \\
\hline $\mathrm{F}_{\mathrm{R}}(\%)$ & 2.7 & 5.3 & 8.4 & 100 \\
\hline
\end{tabular}

$C_{\text {max }}$ : Maximum plasma concentration; DP: Dextran-poly(lactic-co-glycolic acid)-nanoparticle; $F_{R}$ : Relative bioavailability; i.g.: Intragastric administration; LDP: Low molecular weight protamine-dextran-poly(lactic-co-glycolic acid)-nanoparticle; s.c.: Subcutaneous administration ( $n=5$ for all groups); $T_{\max }$ : Time at which $C_{\max }$ is attained.

serum exenatide concentration in the LDP groups was greatly enhanced, being greater than 2.5 -fold higher than that in the exenatide- $\mathrm{Zn}^{2+}$ groups.

\section{Pharmacodynamic study}

Single administration

As shown in Figure 8A, exenatide- $\mathrm{Zn}^{2+}$-DPs induced a maximal blood glucose reduction of $20 \%$ after $6 \mathrm{~h}$, while exenatide- $\mathrm{Zn}^{2+}$-LDPs achieved an impressive hypoglycemic effect at the same time point (70\%). The hypoglycemic 
(A)

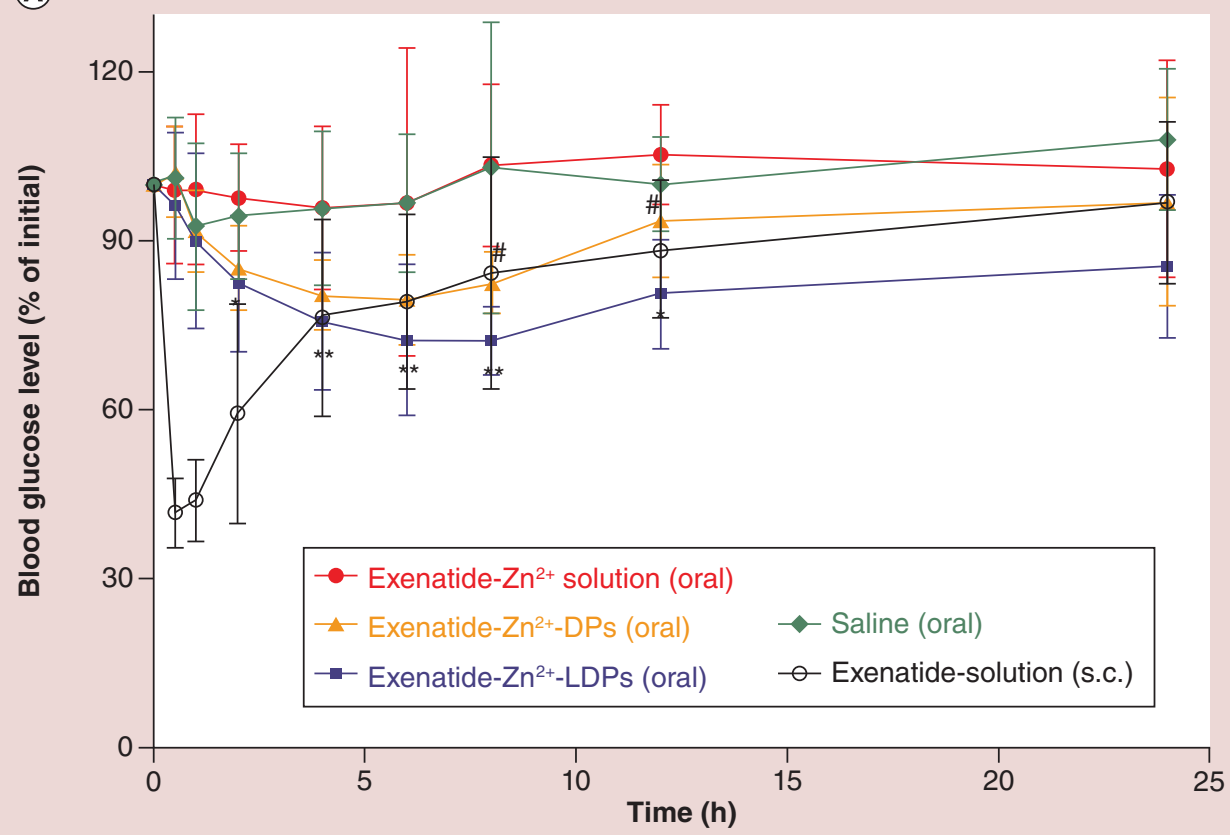

(B)

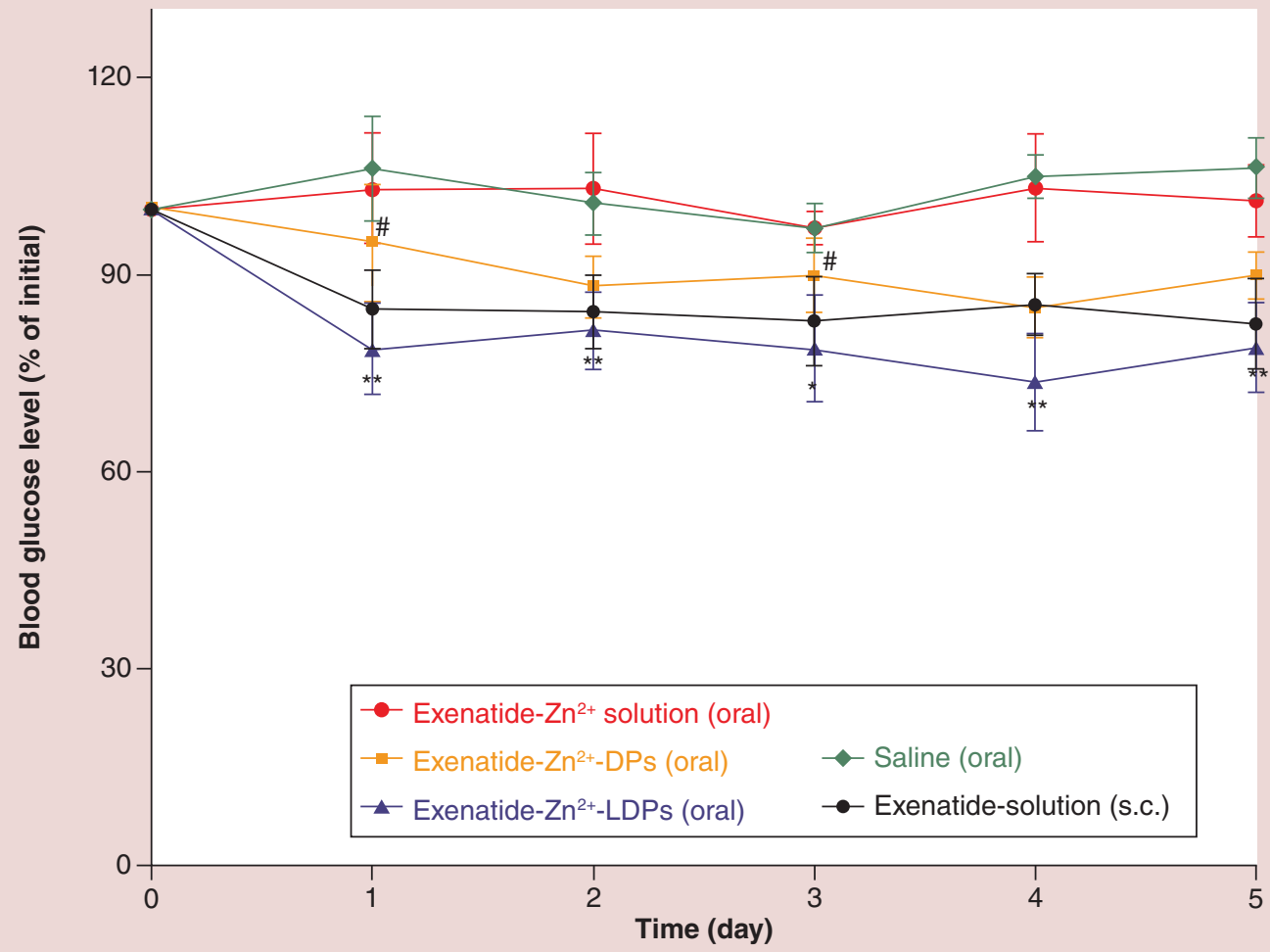

Figure 8. Blood glucose level versus time profles of diabetic rats following the administration of different exenatide formulations. (A) Changes in plasma glucose levels after a single oral administration of saline (control), exenatide-Zn ${ }^{2+}$ solution $(100 \mu \mathrm{g} / \mathrm{kg})$, exenatide-Zn ${ }^{2+}$-DPs $(100 \mu \mathrm{g} / \mathrm{kg})$ or exenatide-Zn ${ }^{2+}$-low molecular weight protamine-dextran-poly(lactic-co-glycolic acid)-nanoparticles $(100 \mu \mathrm{g} / \mathrm{kg})$, or subcutaneous administration of exenatide solution $(10 \mu \mathrm{g} / \mathrm{kg})$ as a positive control. (B) Changes in plasma glucose levels after multiple oral administrations of saline (control), exenatide- $Z n^{2+}$ solution $(100 \mu \mathrm{g} / \mathrm{kg})$, exenatide-Zn ${ }^{2+}-\mathrm{DPs}(100 \mu \mathrm{g} / \mathrm{kg})$ or exenatide-Zn ${ }^{2+}$-low molecular weight protamine-dextran-poly(lactic-co-glycolic acid)-nanoparticles (100 $\left.\mu \mathrm{g} / \mathrm{kg}\right)$, or subcutaneous administration of exenatide solution $(10 \mu \mathrm{g} / \mathrm{kg})$ as a positive control. Data represent means \pm standard deviation $(n=6) .{ }^{*} p<0.05,{ }^{* *} p<0.01$, versus the oral exenatide- $Z n^{2+}$ solution group. $\# p<0.05$, versus the exenatide- $Z \mathrm{n}^{2+}-\mathrm{DPs}$ group.

DP: Dextran-poly(lactic-co-glycolic acid)-nanoparticle; LDP: Low molecular weight protamine-dextran-poly(lactic-co-glycolic acid)-nanoparticle. 
effects after oral administration of exenatide- $\mathrm{Zn}^{2+}$-LDPs showed an extended time compared with subcutaneous injection of exenatide solution.

\section{Multiple administrations}

Using the same format as the single-dose experiment, we conducted repeated administration experiments, as shown in Figure 8B. The blood glucose levels in the LDP, DP and subcutaneous administration groups were all stable, but the LDP groups had the lowest blood glucose levels with decreases to below $80 \%$, as the best hypoglycemic effect.

During the experiment, the dry and wet conditions of the cages in each group were observed every day. It was found that the padding in the subcutaneous groups was always the driest. The paddings in the oral exenatide- $\mathrm{Zn}^{2+}$ solution and saline groups were almost completely wet. The difference between the exenatide- $\mathrm{Zn}^{2+}$-LDP group and the exenatide- $\mathrm{Zn}^{2+}$-DP group was not very large, with about half of the padding wetted with urine. These findings also reflect that oral exenatide- $\mathrm{Zn}^{2+}$-LDPs have a hypoglycemic effect, albeit less effective than that in the subcutaneous group.

\section{Discussion}

Degradation by GI enzymes and poor penetration of the intestinal mucosa are the main obstacles for absorption of peptide drugs $[7,8]$. A variety of strategies to provide structural protection and improve absorption of peptide drugs have been reported. Some of nanocarriers were shown to protect peptides against degradation by intestinal enzymes [11]. However, the development of mucus-diffusive and percutaneous NPs remains a key challenge in the field of oral peptide delivery $[12,13]$. Based on this background, we developed a nanometer carrier that can protect peptide drugs against degradation by GI enzymes, penetrate the mucous layer and be ingested by the intestinal epithelium.

Considering the two main factors of encapsulation efficiency and stability of hydrophilic macromolecular drugs, we decided to use an emulsified solvent volatilization method to prepare the NPs. DEX-PLGA was used as a carrier material to prepare the DPs, and LMWP-DEX-PLGA was used to prepare the LDPs. Table 1 summarizes the characteristics of the blank and drug-loaded NPs. Compared with the blank batch, the drug-loaded DPs had larger particle size and lower $\zeta$-potential, arising from the encapsulation of the negatively charged exenatide resulting in a decrease in the overall potential of the NPs. In addition, both blank LDPs and exenatide- $\mathrm{Zn}^{2+}$-LDPs had lower $\zeta$-potential and larger particle size than DPs. This would be caused by the existence of the negatively charged LMWP. The difference in $\zeta$-potential between DPs and LDPs was not obvious. This may be because the molecular weight of both PLGA and DEX is much higher than that of LMWP, and the electrical properties are dominated by PLGA and DEX. The particle size measured by TEM was obviously smaller than that measured by DLS, possibly because the particle size measured by the DLS instrument also determined the hydration layer around the particles. When sodium phosphotungstate was used for negative staining, it remained in the hydrophilic part of the NPs, thus presenting a deeper black color on the TEM images.

It has been shown that DEX is positively charged, and that its use in nanocrystalline formulations can enhance adhesion to the GI tract [33]. The overall electrically neutral NPs are conducive to the penetration of mucus [13]. The existence of DEX neutralizes the negative electrical charge of PLGA, making the NPs electrically neutral, while LMWP is a long-chain polypeptide that can enhance entanglement between NPs and the mucus layer. The purpose of the next experiment was to verify whether electrically neutral NPs were conducive to penetration of mucus and whether the presence of LMWP hinders mucus penetration. As a precondition for rapid mucus penetration is avoidance of strong entanglement with mucus fibers, we first evaluated the interactions of DPs and LDPs with mucus. The reason why we used DPs and LDPs containing fluorescent substances is that it is difficult to separate high-purity exenatide to determine the concentration of exenatide in the mucus after the experiments, in the case of the use of exenatide DPs or LDPs. As shown in Figure 3A, the relative retention of free FITC-exenatide was about $80 \%$ at $1 \mathrm{~h}$, while that of DPs and LDPs was about $90 \%$. This may be caused by the strong negative charge of free FITC-exenatide. Compared with DPs and LDPs, free FITC-exenatide had a stronger repellent effect for mucus fibers, meaning that its final retention amount was relatively low. In short, the existence of LMWP did not significantly increase the interaction between LDPs and mucus. In addition, the disadvantage of the experiment is that relatively small amounts of NPs or drug solutions are dispersed into relatively large amounts of mucus. At this time, their surface electrical effect is very weak, while a large amount of mucus adhesion is the main. This may also be one of the reasons there is no significant difference among the three groups. 
It was confirmed that the barrier functions of the mucus layer toward DPs, LDPs and free FITC-exenatide were similar. Therefore, the mucus penetration of these three preparations was further investigated. We used a 3D Transwell ${ }^{\circledR}$ system with fresh rat intestinal mucus to better simulate the mucus penetration process. As shown in Figure 3B, the amount of mucus penetration of NPs clearly increased over time. The penetration rate of LDPs was higher than that of DPs and free FITC-exenatide. This was because LDPs were electrically neutral as a whole, and the small amount of LMWP attached to the outer layer of LDPs had a weak negative electronegativity. These two advantages make it easy for LDPs to penetrate the mucilage [34]. In addition, the mucus was negatively charged. Although DPs were electrically neutral, the peripheral DEX had a positive electrical charge. Compared with LDPs, the interaction between DPs and mucus was stronger, thus blocking the penetration of DPs. The experimental results of mucus aggregation and mucus permeation are not contradictory. In the experiment of mucus aggregation, the free drug group is the lowest because of the strong repulsive force between the negative-free drug and the negative mucus. In the mucus permeation experiment, it is also due to the strong negative charge of the free drug, which is not conducive to its penetration. In contrast, NPs are electrically neutral and easily penetrate the mucus layer.

Our previous experiments showed that the presence of LMWP could significantly enhance the intestinal absorption efficiency of LDPs, but the specific mechanism remained unclear. Therefore, we carried out an experiment to clarify the specific uptake mechanism and uptake efficiency of LDPs and DPs. As well as the mucus layer, the intestinal epithelium acts as another obstacle for drug or NP absorption. It has been reported that Caco-2 cell monolayers can be used as an in vitro model of the human intestinal mucosa to predict the absorption of oral drugs or NPs [31]. Therefore, uptake by Caco-2 cells can be used to evaluate the ability of drugs or nanocarriers to enter the intestinal epithelium.

After treatment with coumarin-6-labeled DPs or LDPs, the images of Caco-2 cells were captured by highresolution live cell image system, as shown in Figure 4A. NPs were present in the cytoplasm rather than the nucleus. The fluorescence intensity increased over time, indicating that the uptake of NPs was time dependent. These observations were consistent with those of Zhang et al. [31], who showed that transport of LMWP-NPs across Caco-2 cells was time dependent. Quantitative analyses of the cellular internalization of coumarin-6-labeled DPs and LDPs were performed by flow cytometry (Figure 4B). Interestingly, as observed with the high-resolution live cell imaging system, LDPs exhibited significantly higher counts than DPs, indicating that LMWP promoted the uptake of LDPs. Therefore, highly efficient internalization of the drug was achieved by facilitating by the internalization of LDPs.

The mechanisms for cellular internalization of NPs were investigated by performing uptake experiments under different conditions. The relative amounts of uptake compared with the untreated control group are shown in Figure $6 \mathrm{C} . \mathrm{M}-\beta-\mathrm{CD}$ was applied to investigate the energy-dependent process. The uptake amounts of both DPs and LDPs were reduced in the presence of $M-\beta-C D$, indicating the involvement of an energy-dependent active transport process. $\mathrm{M}-\beta-\mathrm{CD}$ can be an inhibitor of energy-dependent endocytotic processes. Chlorpromazine is a cationic amphiphilic agent that inhibits clathrin-mediated endocytosis by interfering with clathrin adapter protein assembly on the cell surface [35]. After incubation with chlorpromazine, the uptake of LDPs was decreased by about $25 \%$, showing that clathrin was involved in the transport of LDPs. The uptake of LDPs was decreased by about $40 \%$ in formalin-treated cells, indicating that caveolae were involved in LDP transport. The cytotoxicity of endocytosis inhibitors needs to be studied.

The above findings are consistent with those reported by Rejman et al. [17], who suggested that caveolae are more likely to internalize larger particles than clathrin-coated pits. Dos Santos et al. [18] also found that none of the inhibitors used could completely inhibit the uptake of NPs by different cells, and concluded that it was possible to internalize the same NPs in multiple ways at the same time. In addition, protamine was reported to enhance membrane transport [36]. Different mechanisms for this property have been proposed, including the formation of stable hydrogen bonds between basic guanidine groups of arginine and sulfate or phosphate in biofilms. Such interactions are considered to be key factors for membrane transport and therefore increase the cellular uptake of protamine-based nanosystems [37].

To examine whether LMWP can be used as a carrier to improve the absorption efficiency of drugs in the intestinal epithelium, an enterocyte-like model (Caco-2/HT29-MTX co-culture) was employed. Caco-2 and HT29-MTX cells represent the two most abundant cell types in the intestinal epithelium: intestinal epithelial and goblet cells, respectively. Therefore, co-culture of Caco-2 and HT29-MTX cells producing mucus can provide a drug absorption model with a mucus barrier. There was mucus on the surface of the Caco-2/HT29-MTX monolayers. The $\mathrm{P}_{\text {app }}$ was 
used to indicate the ability of each NP type to penetrate the Caco-2 cell monolayers that mimicked small intestinal epithelium. The permeability values of various formulations are given in Table 2 . The Figure 4D indicated that the transmembrane transport of FITC-exenatide was time dependent and the existence of LMWP can improve the transmembrane transport efficiency of LDPs. By comparing the transport capacity of the LDP groups and free LMWP + LDP groups, it was found that the LDP groups had higher capacity than the free LMWP + LDP groups at all time points, indicating that free LMWP possessed some competitive receptor sites that reduced the chance of contact between LDPs and binding sites, thereby reducing the transmembrane transport of LDPs. However, there was no significant difference between the DP groups and the free LMWP + DP groups, indicating that the transport pathway of DPs was different from that of LDPs. It was reported that LMWP has the function of PTD-mediated cell internalization [38].

To observe the fate of orally administered NPs in more detail, we designed an experiment to investigate the distributions of DPs and LDPs in vivo. After oral administration of DiR-labeled LDPs and DPs to mice, both in vivo organs and in vitro isolated organs were observed at various time points. The observations in live mice at different time points are shown in Figure 5A. It can be seen that the fluorescence area increased over time, reflecting the distribution and absorption of the drug in vivo. Compared with the DP group, the fluorescence area in the LDP group expanded more rapidly, indicating that LDP absorption was faster than DP absorption. Figure 5B reveals the fluorescence intensity of isolated organs and indicated that the absorption of LDPs was faster and more widespread than that of DPs [32]. For the DP group, there is some fluorescence around the liver at $0.5 \mathrm{~h}$, but it disappeared at $1 \mathrm{~h}$, which may be caused by contamination during the separation of organs. However, for in vivo experiments, no suitable method has been found to distinguish between diffusion of the stain out of the NPs versus NP penetration. Considering that fluorescent dyes are lipophilic, there was no control experiment to compare the distribution of NPs.

To determine the absorption sites of LDPs and DPs in the intestinal cavity, we investigated the permeability of the intestinal epithelium. The absorption of NPs in this region can be evaluated by the presence or absence of fluorescence, and the absorption efficiency of NPs can be calculated according to the intensity of fluorescence. In this evaluation, the small intestine included the duodenum, jejunum and ileum, and the large intestine included the cecum, colon and rectum. The results of in vivo distribution (Figure 5) showed that the NPs were already absorbed by intestinal epithelium and distributed into various organs after $4 \mathrm{~h}$ of administration. Considering the early contact and absorption of NPs in the upper part of the small intestine, most of the drugs have been absorbed into the systemic circulation for too long after administration, which leads to the weakening of the fluorescence phenomenon in the upper part of the small intestine and affects the observation of the fluorescence localization phenomenon. So the time is set to $150 \mathrm{~min}$. The absorption of LDPs and DPs in the small intestine was higher than that in the large intestine. These findings suggested that drugs and nutrients were mainly absorbed in the small intestine after oral administration. However, the fluorescence intensity of LDPs was stronger than that of DPs in both the large and small intestines, indicating that the presence of LMWP significantly enhanced the absorption efficiency of LDPs in the small intestine. In the large intestine, the fluorescence intensity in the colon was stronger than that in the cecum and rectum. This may be due to the slow rate of material transport in the colon and the large absorption area of the colon arising from the large number of microfolds [39]. As shown in the Figure 6, the fluorescence intensity of the large intestine is weak. In order to determine the difference of absorption in large intestine, semiquantitative method could have been used. If the nuclei had been stained, it would be clear whether the NPs are outside or inside the epithelial cell.

Our ultimate goal for encapsulating therapeutic drugs in NPs is that the drugs can be carried through the mucus layer and across the intestinal epithelial barrier to enter the systemic circulation. Therefore, we investigated the absorption efficiency of NPs in different intestinal segments. For this, we performed in vitro ligation of intestinal loops to examine the intestinal transit volume per unit area of the duodenum, jejunum, ileum and colon. As shown in Figure 6C, the transport capacity of LDPs in the duodenum, jejunum and ileum was about 1.5-times higher than that of DPs, indicating that the presence of LMWP can enhance the absorption efficiency of LDPs in the intestinal epithelium. There was no difference in transport volume between LDPs and DPs in the colon. This may be due to the large absorption of LDPs in the anterior segment of the intestine (mainly the duodenum, jejunum and ileum). When the residual small amount of LDPs arrived at the colon, the concentration of LDPs was very low, and thus the translocation of LDPs per unit area was also low. The results for LDPs and DPs were similar in the colon. Because the concentration of DPs was higher than that of LDPs, the contact rate of DPs per unit area 
was higher than that of LDPs. The control experiments could be used to prove that the loss seen from the interior of the intestinal sacs is caused by the binding and absorption of the NPs by the mucus, or by the permeation.

Bioavailability is an important parameter to determine the pharmacodynamics of peptide drugs. Because of the rapid and complete absorption of drugs after subcutaneous administration, we set the bioavailability of subcutaneous administration to $100 \%$ to calculate the relative bioavailability in the other oral administration groups. The bioavailability in the LDP groups was significantly higher than the DP and solution groups, suggesting that although low bioavailability was observed, LDPs and DPs possessed the capacity to protect a fraction of exenatide against degradation and enhance its intestinal absorption compared with the exenatide- $\mathrm{Zn}^{2+}$ groups. Nonetheless, compared with the oral exenatide- $\mathrm{Zn}^{2+}$ groups, only a slight increase was observed in the DP groups. In contrast, the maximal serum exenatide concentration in the LDP groups was greatly enhanced, being greater than 2.5-fold higher than that in the exenatide- $\mathrm{Zn}^{2+}$ groups. The $\mathrm{T}_{\max }$ of LDPs and DPs is $8 \mathrm{~h}$, which does not indicate that the drug is absorbed in large intestine. Because $T_{\max }$ is the time when the drug's cumulative absorption reaches its $\mathrm{C}_{\max }$, which is consistent with the results of the intestinal epithelium permeability test. Taken together, LDPs successfully optimized the oral delivery of exenatide by inducing higher oral bioavailability of the drug.

Regarding its mechanism of action, exenatide is capable of stimulating $\beta$-cells to release insulin under hyperglycemic conditions, but does not stimulate insulin secretion when the glucose concentration is in the normal range $[40,41]$. Therefore, exenatide cannot promote severe hypoglycemic events. Oral administration of physiological saline had no effect on blood glucose levels. No decrease in the blood glucose level was observed in the exenatide$\mathrm{Zn}^{2+}$ solution groups, indicating that the absorption capacity of free exenatide- $\mathrm{Zn}^{2+}$ in the small intestine was poor. In contrast, subcutaneously injected exenatide solution $(10 \mu \mathrm{g} / \mathrm{kg})$ significantly decreased the glucose level to $44 \%$ of the initial value at $0.25 \mathrm{~h}$. Considering the enzymatic degradation of exenatide in the GI tract and the absorption barrier of the epithelium [13], the dose of exenatide for the NP groups was $100 \mu \mathrm{g} / \mathrm{kg}$. It can be inferred that our NPs delayed the action time and amplified the hypoglycemic efficacy of exenatide. More importantly, LDPs significantly enhanced the in vivo therapeutic efficiency of exenatide, consistent with the results of the in vitro experiments. The hypoglycemic effects after oral administration of exenatide- $\mathrm{Zn}^{2+}$-LDPs showed an extended time compared with subcutaneous injection of exenatide solution. Furthermore, the hypoglycemic effect of the DPs modified by LMWP was much more significant than that of the unmodified DPs at all time points.

Using the same format as the single-dose experiment, we conducted repeated-administration experiments, as shown in Figure $8 \mathrm{~B}$. During the experiment, all of the NPs basically played a hypoglycemic role, and the hypoglycemic effect was obviously improved after three administrations. The reason was that the accumulation of NPs and exenatide in mice increased through a superposition effect of the dosages. The blood glucose levels in the LDP, DP and subcutaneous administration groups were all stable, but the LDP groups had the lowest blood glucose levels with decreases to below $80 \%$, as the best hypoglycemic effect. During the experiment, the dry and wet conditions of the cages in each group were observed every day. These findings also reflect that oral exenatide- $\mathrm{Zn}^{2+}-$ LDPs have a hypoglycemic effect, albeit less effective than that in the subcutaneous group.

\section{Conclusion}

In conclusion, we developed an effective delivery system for oral exenatide NPs that aimed to overcome the multiple intestinal epithelial barriers (especially the mucus barrier and intestinal epithelial cell layer) by using electroneutral LDPs and LMWP PTD-mediated transmembrane transport pathways. Importantly, we further confirmed the absorption sites and absorption rates of LDPs using in vivo imaging systems and confocal laser scanning microscopy. To the best of our knowledge, this was the first time that functional NPs combining electrical neutrality with cell-penetrating peptides have been evaluated for oral delivery of polypeptide drugs. The final in vivo experiments showed that LDPs weakened the blocking effect of mucus and enhanced the affinity for epithelial cells, thus enhancing the cell internalization and transdermal transport efficiency, and also had the effect of reducing blood glucose in Type 2 diabetic mice with a significant and long duration. These findings fully support the research value and significance of the application of electroneutral and cell-penetrating peptide NPs for oral delivery of macromolecular drugs.

\section{Future perspective}

Oral protein and polypeptide drugs are still the focus of pharmaceutical research in the future. The present work is an attempt to combine the surface neutral of NPs with cellular-penetrating peptide for oral delivery. In vivo studies have shown positive results. However, further studies are required to study the optimal LMWP modification 
amount of block polymer LMWP-DEX-PLGA. In addition, the effects or mechanisms of LDPs and DPs on the tight junctions between cells and the transport pathway of $\mathrm{M}$ cells are also indispensable for the development of oral delivery systems for nanopharmaceuticals. The cytotoxicity of NPs and endocytosis inhibitors remains to be further clarified. It may be expected that more and more oral drug-delivery systems will be developed in the future, which focus not only on overcoming GI acid-base environment and digestive enzyme degradation, but also on improving the targeted absorption efficiency of intestinal epithelium.

\section{Financial \& competing interests disclosure}

This work was supported by the Natural Science Foundation of Shandong (No. ZR2014HM062). The authors have no other relevant affiliations or financial involvement with any organization or entity with a financial interest in or financial conflict with the subject matter or materials discussed in the manuscript apart from those disclosed.

The authors thank A Sherwin, from Liwen Bianji, Edanz Group China (www.liwenbianji.cn/ac) for editing the English text of a draft of this manuscript.

\section{Ethical conduct of research}

All animal experiments were approved by the Experimental Animal Ethics Committee of the School of Pharmacy, Yantai University. The authors state that they have obtained appropriate institutional review board approval or have followed the principles outlined in the Declaration of Helsinki for all human or animal experimental investigations.

\section{Open access}

This work is licensed under the Attribution-NonCommercial-NoDerivatives 4.0 Unported License. To view a copy of this license, visit http://creativecommons.org/licenses/by-nc-nd/4.0/

\section{Summary points}

Characterization of low molecular weight protamine-dextran-poly(lactic-co-glycolic acid)-nanoparticles

- The particle size of exenatide-Zn ${ }^{2+}$-low molecular weight protamine-dextran-poly(lactic-co-glycolic acid)-nanoparticles (LDPs) was $141.1 \pm 6.9 \mathrm{~nm}$ and the $\zeta$-potential was $0.16 \pm 0.027 \mathrm{mV}$.

- The LDPs were uniformly spherical.

Study on aggregation \& permeability of mucus

- The existence of low molecular weight protamine (LMWP) did not significantly increase the interaction between LDPs and mucus.

- Because LDPs were electrically neutral as a whole, and the small amount of LMWP attached to the outer layer of LDPs had weak negative electronegativity, these two advantages allowed LDPs to easily penetrate the mucilage.

Distribution of LDPs in vivo

- The fluorescence area in the LDP group expanded faster than that in the dextran-poly(lactic-co-glycolic acid)-nanoparticle (DP) group, indicating that LDP absorption was faster than DP absorption.

- Compared with the DP group mice, the organs in the LDP group mice had earlier fluorescence and stronger fluorescence intensity.

Permeability of the intestinal epithelium

- The transport capacity of LDPs in the duodenum, jejunum and ileum was higher than that of DPs, showing that the presence of LMWP can enhance the absorption efficiency of the intestinal epithelium toward LDPs.

Cellular experiments

- Highly efficient internalization of the drug was achieved by facilitating by the internalization of LDPs.

- Regarding the mechanisms for cellular internalization, LDPs exhibited an energy-dependent active transport process that involved clathrin- and caveolae-mediated endocytosis.

- The transepithelial transport studies demonstrated that LMWP had the function of protein transduction domain-mediated cell internalization.

In vivo pharmacokinetic studies

- LDPs successfully optimized oral delivery of exenatide by inducing higher oral bioavailability of the drug.

Pharmacodynamic study

- The hypoglycemic effect of LDPs was much more significant than that of unmodified DPs at all time points. 


\section{References}

Papers of special note have been highlighted as: $\bullet$ of interest; $\bullet \bullet$ of considerable interest

1. Hu X, Fan W, Yu Z et al. Evidence does not support absorption of intact solid lipid nanoparticles via oral delivery. Nanoscale 8(13), 7024-7035 (2016).

2. Malhaire H, Gimel JC, Roger E, Benoit JP, Lagarce F. How to design the surface of peptide-loaded nanoparticles for efficient oral bioavailability? Adv. Drug Deliv. Rev. 106(Pt B), 320-336 (2016).

3. Aguirre TA, Teijeiro-Osorio D, Rosa M, Coulter IS, Alonso MJ, Brayden DJ. Current status of selected oral peptide technologies in advanced preclinical development and in clinical trials. Adv. Drug Deliv. Rev. 106(Pt B), 223-241 (2016).

- Advantages of oral polypeptide nanoparticles in the treatment of diabetes mellitus.

4. Bouttefeux O, Beloqui A, Preat V. Delivery of peptides via the oral route: diabetes treatment by peptide-loaded nanoparticles. Curr. Pharm. Des. 22(9), 1161-1176 (2016).

5. Maher S, Mrsny RJ, Brayden DJ. Intestinal permeation enhancers for oral peptide delivery. Adv. Drug Deliv. Rev. 106(Pt B), 277-319 (2016).

6. Li X, Yu M, Fan W, Gan Y, Hovgaard L, Yang M. Orally active-targeted drug delivery systems for proteins and peptides. Expert Opin. Drug Deliv. 11(9), 1435-1447 (2014).

7. Groo AC, Saulnier P, Gimel JC et al. Fate of paclitaxel lipid nanocapsules in intestinal mucus in view of their oral delivery. Int. J. Nanomedicine 8, 4291-4302 (2013).

8. Smart AL, Gaisford S, Basit AW. Oral peptide and protein delivery: intestinal obstacles and commercial prospects. Expert Opin. Drug Deliv. 11(8), 1323-1335 (2014).

9. Su FY, Lin KJ, Sonaje K et al. Protease inhibition and absorption enhancement by functional nanoparticles for effective oral insulin delivery. Biomaterials 33(9), 2801-2811 (2012).

10. Trapani A, Laquintana V, Denora N et al. Eudragit RS 100 microparticles containing 2-hydroxypropyl- $\beta$-cyclodextrin and glutathione: physicochemical characterization, drug release and transport studies. Eur. J. Pharm. Sci. 30(1), 64-74 (2007).

11. Bakhru SH, Furtado S, Morello AP, Mathiowitz E. Oral delivery of proteins by biodegradable nanoparticles. Adv. Drug Deliv. Rev. 65(6), 811-821 (2013).

12. Dunnhaupt S, Kammona O, Waldner C, Kiparissides C, Bernkop-Schnurch A. Nano-carrier systems: strategies to overcome the mucus gel barrier. Eur. J. Pharm. Biopharm. 96, 447-453 (2015).

-. The opportunities and limitations of peptides and nanoparticles in the gastrointestinal after oral administration.

13. Lundquist P, Artursson P. Oral absorption of peptides and nanoparticles across the human intestine: opportunities, limitations and studies in human tissues. Adv. Drug Deliv. Rev. 106(Pt B), 256-276 (2016).

-• Exenatide- $\mathrm{Zn}(2+)$ complex enhances the bioactivity of exenatide by oral administration.

14. Zhang L, Shi Y, Song Y et al. Tf ligand-receptor-mediated exenatide- $\mathrm{Zn}(2+)$ complex oral-delivery system for penetration enhancement of exenatide. J. Drug Target. 26, 1-10 (2018).

15. Lisi GP, Hughes RP, Wilcox DE. Coordination contributions to protein stability in metal-substituted carbonic anhydrase. J. Biol. Inorg. Chem. 21(5-6), 659-667 (2016).

16. Niesner U, Halin C, Lozzi L et al. Quantitation of the tumor-targeting properties of antibody fragments conjugated to cell-permeating HIV-1 TAT peptides. Bioconjug. Chem. 13(4), 729-736 (2002).

17. Rejman J, Oberle V, Zuhorn IS, Hoekstra D. Size-dependent internalization of particles via the pathways of clathrin- and caveolae-mediated endocytosis. Biochem. J. 377(Pt 1), 159-169 (2004).

- Study on the mechanism of interaction between protamine and small intestinal epithelium.

18. dos Santos T, Varela J, Lynch I, Salvati A, Dawson KA. Effects of transport inhibitors on the cellular uptake of carboxylated polystyrene nanoparticles in different cell lines. PLoS ONE 6(9), e24438 (2011).

19. Schwarze SR, Ho A, Vocero-Akbani A, Dowdy SF. In vivo protein transduction: delivery of a biologically active protein into the mouse. Science 285(5433), 1569-1572 (1999).

20. Chalasani KB, Russell-Jones GJ, Yandrapu SK, Diwan PV, Jain SK. A novel vitamin B12-nanosphere conjugate carrier system for peroral delivery of insulin. J. Control. Rel. 117(3), 421-429 (2007).

21. Kallinteri P, Higgins S, Hutcheon GA, St Pourcain CB, Garnett MC. Novel functionalized biodegradable polymers for nanoparticle drug delivery systems. Biomacromolecules 6(4), 1885-1894 (2005).

22. Chalasani KB, Russell-Jones GJ, Jain AK, Diwan PV, Jain SK. Effective oral delivery of insulin in animal models using vitamin B12-coated dextran nanoparticles. J. Control. Rel. 122(2), 141-150 (2007).

23. Kaklotar D, Agrawal P, Abdulla A et al. Transition from passive to active targeting of oral insulin nanomedicines: enhancement in bioavailability and glycemic control in diabetes. Nanomedicine 11(11), 1465-1486 (2016).

24. He J, Li H, Liu C et al. Formulation and evaluation of poly(lactic-co-glycolic acid) microspheres loaded with an altered collagen type II peptide for the treatment of rheumatoid arthritis. J. Microencapsul. 32(6), 608-617 (2015). 
25. Yuan H, Chen CY, Chai GH, Du YZ, Hu FQ. Improved transport and absorption through gastrointestinal tract by PEGylated solid lipid nanoparticles. Mol. Pharm. 10(5), 1865-1873 (2013).

26. Zhang X, Sun M, Zheng A, Cao D, Bi Y, Sun J. Preparation and characterization of insulin-loaded bioadhesive PLGA nanoparticles for oral administration. Eur. J. Pharm. Sci. 45(5), 632-638 (2012).

27. Sood A, Panchagnula R. Peroral route: an opportunity for protein and peptide drug delivery. Chem. Rev. 101(11), 3275-3303 (2001).

28. Kiyono H, Fukuyama S. NALT-versus Peyer's-patch-mediated mucosal immunity. Nat. Rev. Immunol. 4(9), 699-710 (2004).

- In vitro and in vivo studies on the membrane translocation effects of low molecular weight protamine on polypeptide delivery.

29. Park YJ, Chang LC, Liang JF, Moon C, Chung CP, Yang VC. Nontoxic membrane translocation peptide from protamine, low molecular weight protamine (LMWP), for enhanced intracellular protein delivery: in vitro and in vivo study. FASEB J. 19(11), 1555-1557 (2005).

30. Shan W, Zhu X, Tao W et al. Enhanced oral delivery of protein drugs using zwitterion-functionalized nanoparticles to overcome both the diffusion and absorption barriers. ACS Appl. Mater. Interfaces 8(38), 25444-25453 (2016).

-• Preparation of functional targeting nanoparticles for oral delivery of biomolecules.

31. Zhang L, Shi Y, Song Y et al. The use of low molecular weight protamine to enhance oral absorption of exenatide. Int. J. Pharm. 547(1-2), 265-273 (2018).

32. Wu L, Liu M, Shan W et al. Bioinspired butyrate-functionalized nanovehicles for targeted oral delivery of biomacromolecular drugs. J. Control. Rel. 262, 273-283 (2017).

-• Study on oral administration of insulin nanoparticles prepared by dextran-poly(lactic-co-glycolic acid) polymer.

33. Alibolandi M, Alabdollah F, Sadeghi F et al. Dextran-b-poly(lactide-co-glycolide) polymersome for oral delivery of insulin: in vitro and in vivo evaluation. J. Control. Rel. 227, 58-70 (2016).

34. Zhang P, Xu Y, Zhu X, Huang Y. Goblet cell targeting nanoparticle containing drug-loaded micelle cores for oral delivery of insulin. Int. J. Pharm. 496(2), 993-1005 (2015).

35. Zhang J, Zhu X, Jin Y, Shan W, Huang Y. Mechanism study of cellular uptake and tight junction opening mediated by goblet cell-specific trimethyl chitosan nanoparticles. Mol. Pharm. 11(5), 1520-1532 (2014).

36. Kristensen M, Birch D, Morck Nielsen H. Applications and challenges for use of cell-penetrating peptides as delivery vectors for peptide and protein cargos. Int. J. Mol. Sci. 17(2), (2016).

37. He B, Lin P, Jia Z et al. The transport mechanisms of polymer nanoparticles in Caco-2 epithelial cells. Biomaterials 34(25), 6082-6098 (2013).

38. Thwala LN, Beloqui A, Csaba NS et al. The interaction of protamine nanocapsules with the intestinal epithelium: a mechanistic approach. J. Control. Rel. 243, 109-120 (2016).

39. Molteni L. Dextran and inulin conjugates as drug carriers. Methods Enzymol. 112, 285-298 (1985).

40. Awaad A, Nakamura M, Ishimura K. Imaging of size-dependent uptake and identification of novel pathways in mouse Peyer's patches using fluorescent organosilica particles. Nanomedicine 8(5), 627-636 (2012).

41. Shi Y, Sun X, Zhang L et al. Fc-modified exenatide-loaded nanoparticles for oral delivery to improve hypoglycemic effects in mice. Sci. Rep. 8(1), 726 (2018). 
